\title{
Astrophysical dust formation under the influence of temperature fluctuations
}

\section{A general stochastic approach and its application to AGB winds}

\author{
U. Dirks, V. Schirrmacher, and E. Sedlmayr
}

\begin{abstract}
Zentrum für Astronomie und Astrophysik (ZAA), TU Berlin, Hardenbergstr. 36, 10623 Berlin, Germany
\end{abstract} e-mail: schirrmacher@astro.physik.tu-berlin.de

Received 13 December 2007 / Accepted 9 July 2008

\section{ABSTRACT}

\begin{abstract}
Context. Dust formation, i.e. the condensation of solid particles from a gas phase, occurs in astrophysical environments that are sufficiently cool and offer a sufficiently high density of condensable species. Since many astrophysical systems in which dust forms have turbulent features and the dust formation rates depend significantly on temperature, we investigate the influence of temperature fluctuations on dust condensation in dust-driven AGB winds.

Aims. We present an approach for the time-dependent stochastic description of astrophysical dust formation and apply this in developing a fast algorithm, which is well suited for implementation into numerical models of dynamical AGB winds. The influence of the fluctuations on the dynamical structure is then investigated for a large sample of wind parameters.

Methods. We employ a mathematical method, which describes the dust formation under the influence of random temperature fluctuations as a stochastic process. A system of Fokker-Planck equations for the one-point distribution function of the conjoint temporal development of temperature and dust results. Gas-box studies of this system have led us to a simplified microturbulent description for the implementation into time-dependent numerical wind models.

Results. A method for the investigation of temperature fluctuations on dust formation is presented, which can be adapted to a variety of astrophysical situations. For dust-driven AGB winds, a microturbulent description turns out to be admissible. In AGB winds, rmstemperature fluctuations of 20-60 K can result in increased mass loss rates of 10-30\% depending on the details of the physical model assumptions.

Conclusions. The presented method for the treatment of temperature fluctuations with respect to dust formation has been succesfully applied to dynamical AGB winds. We suggest how to apply the method to other astrophysical systems. The influence of the fluctuations on the dynamics of dust-driven AGB winds is remarkable, but not overwhelming.
\end{abstract}

Key words. turbulence - stars: mass-loss - stars: AGB and post-AGB - stars: winds, outflows - methods: statistical

\section{Introduction}

Dust is an ubiquitous phenomenon in our Galaxy. It is an effective absorber of radiation at short wavelengths and re-emits thermal IR-radiation. Due to this strong interaction between dust and radiation fields, astrophysical situations in which dust formation occurs, often show highly non-linear behaviour in the sense that not only the energy balance of the systems often switches between a dust-free and a dust-enshrouded situation, but also the momentum balance is affected severely by the radiation pressure onto the absorbing dust, which can induce hydrodynamic motions and even trigger stellar winds.

Astrophysical dust-forming systems are often affected by fluctuating thermodynamic conditions. The origin of these fluctuations could be (magneto)-hydrodynamical waves, turbulence during the dissipation of momentum input by a stellar pulsation (AGB stars), eruption (novae, R Coronae Borealis stars), explosion (supernovae), or even convection (in the atmospheres of Brown Dwarfs or gas planets) - see e.g. Woitke (2006) for AGB stars or Helling et al. (2001), and Helling \& Woitke (2006) for Brown Dwarfs.

Numerical model calculations are an important tool in understanding the physics of dust-driven winds of AGB stars and in interpretating observations (see e.g. Bowen 1988; Dorfi \& Höfner 1991; Fleischer et al. 1992; Winters et al. 1997;
Schirrmacher et al. 2003; Jeong et al. 2003; Gautschy-Loidl et al. 2004; Woitke 2006). Although the stellar pulsation originates in the turbulent convective stellar envelope, the influence of temperature fluctuations on the dust condensation in these objects has never been included in numerical model calculations of AGB star winds, before this study. It could be argued that the effects of fluctuations on spatial or temporal scales smaller than the numerical resolution will cancel out because fluctuations at higher temperatures will be balanced by fluctuations at lower temperatures. By ignoring the effects of sub-scale fluctuations, we would still obtain accurate mean values of physical quantities. However, this is only the case for processes that react symmetrically onto temperature fluctuations.

The dust nucleation, in turn, reacts asymmetrically to temperature fluctuations, because a seed formed during a temporary temperature decrease does not necessarily evaporate during a subsequent temperature rise. A high supersaturation is required for dust nucleation, whereas once a particle has grown to macroscopic size, it only begins to re-evaporate when the supersaturation ratio drops below 1 . Due to this asymmetric response of the dust nucleation to temperature fluctuations, situations might occur where a fluctuating medium shows dust condensation, despite the fact that the mean thermodynamic conditions would not allow dust nucleation. Since in this case, the effect of even symmetric fluctuations on a sub-grid level will not cancel out, a 
sub-grid model is required, to take into account the influence of the fluctuations on the dust condensation. In this work, we therefore, develop a general method for taking into account the influence of temperature fluctuations on dust formation, and apply this to the dust-driven winds of AGB stars.

The development of advanced models for the two-stage transition from the gas phase to a solid body in which small metastable nucleation seeds emerge such as clusters, which grow under appropriate temperature conditions to become macroscopic particles, (see Gail et al. 1984; Gail \& Sedlmayr 1988; Gauger et al. 1990; Patzer et al. 1998), allowed the deterministic calculation of dust-driven AGB winds (see e.g. Fleischer et al. 1992; Höfner et al. 1996; Schirrmacher et al. 2003). However, AGB stars have convective turbulent stellar envelopes, in which the stellar pulsations are generated and fluctuating thermodynamic conditions must be expected. Since no quantitative information about the strength of the related temperature fluctuations is at hand, we treat the root mean square (rms) temperature deviation $\sigma$ and the correlation time $\lambda$ as free parameters and investigate their influence on dust formation.

Our approach is based on a phenomenological, statistical description of the stochastic temperature fluctuations. The approach is based on the procedures developed by Gail et al. (1974), Gail \& Sedlmayr (1974), and Gail et al. (1980). To be able to clarify and generalize the mathematic modelling, we use the theory of stochastic processes and stochastic differential equations in our investigation. We derive a system of FokkerPlanck equations for the one-point distribution function, which indicates the joint probability of a certain state of dust formation and temperature at a given time.

This Fokker-Planck system was solved in a gas box for a typical trajectory of a volume element in a stellar wind, to determine how strong the temperature fluctuations had to be to influence the dust condensation in an AGB wind significantly, and on what timescales the stochastic condensation process can be modelled in the microturbulent limit case. A microturbulent description was found to be admissible for correlations times lower than $10^{4} \mathrm{~s}$ and rms-temperature deviations even as small as $20 \mathrm{~K}$ had a remarkable influence on the condensation process. Since the longest time steps in the dynamical code used in this work are of the order of several $10^{3} \mathrm{~s}$, this means that all fluctuations occuring on a sub-grid scale can be modelled by the microturbulent limit case. Therefore, in this work, a one-parametric microturbulent description of the stochastical dust condensation is developed and implemented into the CHILD-Code, a dynamical 1D-code that self-consistently solves the equations of hydrodynamics, thermodynamics, and time-dependent dust condensation, including a grey radiative transfer and an equilibrium chemistry for the species that are most important to dust condensation. The implementation of the presented sub-grid model into other numerical models for AGB winds, such as multidimensional models (e.g. Woitke 2006) or models including a frequency-dependent radiative transfer (e.g. Gautschy-Loidl et al. 2004), should be straightforward.

This paper develops a methodological approach to a theory of dust formation and growth under stochastic temperature fluctuations, introduced by Dirks (2000), with preliminary studies by Dirks (1993), Dirks \& Sedlmayr (1998), and the studies of Schirrmacher \& Dirks (2003), Schirrmacher et al. (2004) and Schirrmacher et al. (2005), and continues with the quantitative investigations of dust formation by Schirrmacher (2007).

In Sect. 2, we describe the deterministic dust formation theory, upon which this work is based. In Sect. 3, we describe our stochastical model for the temperature fluctuations and derive a system of Fokker-Planck equations, which describes dust formation under the influence of temperature fluctuations. In Sect. 4, we present a set of gas box calculations, where the influence of the fluctuation parameters in an AGB-like environment is investigated. The derivation of a fast microturbulent approach and its implementation into a self-consistent dynamical code for AGB winds, and our results and discussion are presented in Sect. 5. Our summary, conclusions, and perspective on future work are provided in Sect. 6.

\section{Dust formation, grain growth, and evaporation for deterministic conditions}

We describe the dust component by a grainsize distribution function. This choice restricts our study to homogeneous dust grains, which consist of a number of identical monomers. However, growth processes including several growth species can be considered, provided that they result in the addition of an $i$-mer to the grain. Since the focus of this work is on the general impact of fluctuations on the condensation process and not on the detailed chemical composition of the grains, this approach seems adequate. We consider carbon grains with $\mathrm{C}, \mathrm{C}_{2}, \mathrm{C}_{2} \mathrm{H}$, and $\mathrm{C}_{2} \mathrm{H}_{2}$ as growth species.

\subsection{Dust description}

We apply the moment method developed by Gail et al. (1984), Gail \& Sedlmayr (1988), and Gauger et al. (1990), which provides a set of equations for the moments $K_{\mu}$ of the grain size distribution function $f(N, t)$ containing the number density ${ }^{1}$ of dust grains of size $N$ (= number of monomers contained) at any given $t$. The moments $K_{\mu}$ of $f(N, t)$ are defined by:

$K_{\mu}(t)=\sum_{N=N_{l}}^{N_{\max }} N^{\mu / d} f(N, t) \quad(\mu=0,1,2, \ldots)$.

$N_{l}$ is the minimum grain size, i.e. an arbitrary border between gas and solid phase ${ }^{2}$, and $d$ is the spatial dimension of the grains, which by assuming spherical particles we set to be $d=3$ in this work. The first four moments have an immediate physical interpretation:

1. particle density $n_{\mathrm{d}}$ of grains of size $\geq N_{\ell}$ :

$n_{\mathrm{d}}=K_{0}$

2. average grain radius:

$$
\langle r\rangle=r_{0} \frac{K_{1}}{K_{0}},
$$

where $r_{0}$ is the (hypothetical) radius of a monomer; 3. average grain surface:

$$
\left\langle A_{\mathrm{N}}\right\rangle=4 \pi r_{0}^{2} \frac{K_{2}}{K_{0}}
$$

${ }_{1}$ In this work, all densities are scaled to the number density of hydrogen cores $n_{\hat{\mathrm{H}}}$.

${ }^{2} N_{l}$ has to be chosen larger than the largest $i$-mer, that is expected to contribute to the growth process, so that all growth species belong to the gas phase. Otherwise the grain density would be lowered by growth. We note that for this reason coagulation processes cannot be treated in the framework of this method. 
4. average particle size:

$$
\langle N\rangle=\frac{K_{3}}{K_{0}}
$$

5. number density $n_{\mathrm{c}}$ of monomers condensed into grains of size $\geq N_{\ell}$ :

$$
n_{\mathrm{c}}=K_{3} \text {. }
$$

Thus, $K_{3}$ is a measure of the consumption of monomers from the gas phase and must be taken into account when calculating the carbon abundance $\epsilon_{\mathrm{C}}$

$\epsilon_{\mathrm{C}}(t)=\epsilon_{\mathrm{C}, 0}-K_{3}(t)$,

where $\epsilon_{\mathrm{C}, 0}$ is the undepleted carbon abundance of the dust free gas. Formation, growth, and evaporation of the dust are described by a two-step process: first, seed particles need to be formed, and subsequently, these particles grow by capturing more monomers from the gas phase. The formation of seed particles is assumed to be given by the stationary nucleation rate $J_{*}$, calculated according to classical nucleation theory (see Gail et al. 1984, and references therein), whereas the net growth of the particles is assumed to occur by homogeneous growth, chemical growth, thermal evaporation, and chemical sputtering as calculated by Gail \& Sedlmayr (1988) and Gauger et al. (1990).

The time evolution in the first four dust moments is then given by (see Gauger et al. 1990)

$$
\begin{aligned}
\frac{\mathrm{d} K_{0}}{\mathrm{~d} t} & =J_{N_{\ell}}, \\
\frac{\mathrm{d} K_{\mu}}{\mathrm{d} t} & =N_{\ell} \frac{\mu}{d} J_{N_{\ell}}+\frac{\mu}{\mathrm{d}} \frac{1}{\tau} K_{\mu-1} \quad(\mu=1,2,3),
\end{aligned}
$$

where $J_{N_{\ell}}$ is the creation/destruction rate of clusters of size $N_{\ell}$. It is assumed that $J_{N_{\ell}}$ corresponds to the stationary nucleation rate $J_{*}$, and $\tau^{-1}$ corresponds to the net growth rate, i.e. including the effects of dust destruction.

Mathematically, Eq. (4a) is a closing condition for the equation system Eq. (4b), so that the moments with negative $\mu$ do not have to be evaluated.

We introduce

$\mathrm{A}=\left(\begin{array}{cccc}0 & 0 & 0 & 0 \\ \frac{1}{3} \tau^{-1} & 0 & 0 & 0 \\ 0 & \frac{2}{3} \tau^{-1} & 0 & 0 \\ 0 & 0 & \tau^{-1} & 0\end{array}\right), \boldsymbol{b}=\left(\begin{array}{c}J_{\mathrm{N}_{\ell}} \\ N_{\ell}^{\frac{1}{3}} J_{\mathrm{N}_{\ell}} \\ N_{\ell}^{\frac{2}{3}} J_{\mathrm{N}_{\ell}} \\ N_{\ell} J_{\mathrm{N}_{\ell}}\end{array}\right)$,

and $\boldsymbol{K}=\left(\begin{array}{c}K_{0} \\ K_{1} \\ K_{2} \\ K_{3}\end{array}\right)$

The equation system Eq. (4) can then be written as:

$\frac{\mathrm{d}}{\mathrm{d} t} \boldsymbol{K}=\mathrm{A} \cdot \boldsymbol{K}+\boldsymbol{b}$.

\subsection{Modelling the environment for the dust calculation}

The moment method presented in the previous section can be applied to all astrophysical systems in which dust formation and growth occur in a previously dust-free environment. The nucleation rate $J$ and net growth rate $\tau^{-1}$ are functions of the gas temperature $T_{\mathrm{g}}$, the dust temperature $T_{\mathrm{d}}$, and the particle densities $n_{i}$ of the growth species.
When adapting this method to various astrophysical environments, the time-dependent temperature und density structure must be provided, including the chemical particle densities of the growth species at any moment in time. Obviously, the particle densities of the growth species depend on the degree of condensation (see Eq. (3)). The temperature structure of the object usually also depends on the spatio-temporal dust distribution, because dust tends to dominate the energy exchange between a gas/dust mixture and any given radiation field. In dust-driven winds, the radiation pressure on the dust will be a dominant input term in the calculation of the hydrodynamical structure, i.e. the density profile.

The equation system Eq. (5) will conveniently become part of the Fokker-Planck system Eq. (34) derived in Sect. 3. It will therefore remain necessary to provide the density, temperature, and particle densities of all growth species. Since a coupled solution of the probabilistic set of equations and hydrodynamic equations with radiative transfer ${ }^{3}$ would considerably increase the computation time, we first performed some gas box studies, in which we neglected the coupling to the hydrodynamic structure and radiative transfer, and calculated the probabilistic dust formation on a prototype, non-varying wind model described in Sect. 4. The results of these gas box studies lead us to a microturbulent approach, which is suitable for the implementation into the self-consistent dynamical wind calculations presented in Sect. 5 .

\section{Stochastic model}

Since we have neither a concrete turbulence theory of stellar atmospheres, nor an observation-based quantitative knowledge concerning the irregular fluctuations, they must be analyzed using a statistical description, which accounts satisfactorily for fluctuations phenomenologically but does not include explicitly their physical causes.

The following approach therefore represents a formulation of the physics of dust formation in terms of stochastic processes, and a mathematical generalisation of physical problem constitutions as developed in the domain of line formation in random velocity fields, in particular by Gail et al. (1974), Gail \& Sedlmayr (1974), and Gail et al. (1980). Within the concrete implementation, the fluctuation effect is limited to the temperature parameter without any restriction of the generality of this method, inasmuch as temperature is the most influential physical parameter with regard to dust formation and growth.

Further details of this approach and its mathematical background are given by Dirks (2000).

\subsection{Dust formation as a stochastic process}

Within a stellar wind of velocity $\boldsymbol{v}$, we describe generally field quantities $X$ in a gas volume element traveling radially towards the periphery of the circumstellar envelope, which at time $t_{0}=0$ is situated at the surface of the $\operatorname{star} r=R_{*}$, by indicating the state $X=X(t)$ within the comoving system. The temperature evolution can be described by a fluctuation conditional temperature deviation by the splitting of $T_{\mathrm{g}}(t)$ into $\bar{T}(t)+T(t)$, where $\bar{T}(t)$ denotes the deterministic mean value of the temperature and $T(t)$ the stochastic temperature deviation.

\footnotetext{
3 In the deterministic case, these calculations were performed by e.g. Fleischer et al. (1992), Sedlmayr \& Winters (1997), Winters et al. (2000), and Schirrmacher et al. (2003).
} 
As is customary in probability theory, we consider, in the framework of a statistical model, the determination (measurement) of the temperature deviation at time $t$ in the gas element to be the random variable $\Theta_{t}$. Because of the significant dependence of both dust formation and growth on temperature, their probabilistic state is described by a random variable $\mathcal{K}$ for the determination of the values of the dust moments $\hat{K}_{0}, \ldots, \hat{K}_{3}$. The evolution in both temperature and dust, in the state space $\left(\mathbb{R}^{5}, \mathcal{B}^{5}\right)$ at each time, will therefore be described by the family of random variables, given by the time parameter $t$, i.e. by the stochastic process $\left\{(\Theta, \mathcal{K})_{t} ; t \in \mathbb{R}_{0}^{+}\right\}$, the paths of which represent the possible evolutions

$(\Theta, \mathcal{K})_{t}(T, \hat{\boldsymbol{K}})=\left(T, \hat{K}_{0}, \hat{K}_{1}, \hat{K}_{2}, \hat{K}_{3}\right)(t) ;$

in this context, $\mathcal{B}$ designates the Borel sets of $\mathbb{R}$.

In view of the physical causes of the temperature fluctuations, it is justified to assume the independence of the temperature fluctuations and the present dust growth from the former temperature fluctuations as well as the concrete genesis of the given dust particles. In that sense, the stochastic process is "without memory", that is, for a given present state, both the past and future of its physical development are statistically independent of each other. With regard to the component of dust formation and growth in itself, this process is indeed described by common differential equations of first order (Eqs. (4a,b)), i.e. by a functional connection in which the change in the system at time $t$ depends only on the state at this time, and not on former states. Altogether, we postulate that the stochastic process $\left\{(\Theta, \mathcal{K})_{t} ; t \in \mathbb{R}_{0}^{+}\right\}$is a Markov process.

In terms of the fundamental theorem of Kolmogorov (see e.g. Arnold 1974, Theorem 1.8.1), a stochastic process is canonically given by a specified consistent family of finite-dimensional distributions, apart from stochastic equivalence. As for Markov processes, the finite-dimensional distributions are determined by indicating a start distribution and a transition probability, and the construction of the searched process is simplified by assuming the Markov-presupposition (see Arnold 1974, Chap. 2).

In the present case, we must determine the one-point probability distribution to calculate the expectation values for dust formation during temperature fluctuations; under the physically justified assumption of the continuous existence of probability densities, we therefore search for the density $p_{1}(t, T, \hat{\boldsymbol{K}})$, which, in the form

$p_{1}(t, T, \hat{\boldsymbol{K}}) \Delta T \Delta \hat{K}_{0} \cdots \Delta \hat{K}_{3}$

for sufficiently small $\Delta T$ and $\Delta \hat{K}_{\mu}$, indicates the probability that the fluctuation temperature reaches a value in the range $[T, T+\Delta T]$ and the dust formation moments reach a value in the range $\left[\hat{K}_{\mu}, \hat{K}_{\mu}+\Delta \hat{K}_{\mu}\right]$. The stochastic information about this Markovian process from the transition probability density

$p\left(t, T, \hat{\boldsymbol{K}}, t+\Delta t, T^{\prime}, \hat{\mathbf{K}}^{\prime}\right)$

indicates the probability that the process is found in the state $\left(T^{\prime}, \hat{\mathbf{K}}^{\prime}\right)$ at time $t+\Delta t$, if it was found in the state $(T, \hat{\boldsymbol{K}})$ at the previous time $t$. The Chapman-Kolmogorov equation (also known as Einstein-Smoluchowski equation)

$$
\begin{aligned}
p\left(t_{1}, T_{1}, \hat{\boldsymbol{K}}_{1}, t_{2}, T_{2}, \hat{\boldsymbol{K}}_{2}\right)= & \int p\left(t_{1}, T_{1}, \hat{\boldsymbol{K}}_{1}, t, T, \hat{\boldsymbol{K}}\right) \\
& \cdot p\left(t, T, \hat{\boldsymbol{K}}, t_{2}, T_{2}, \hat{\boldsymbol{K}}_{2}\right) \mathrm{d} T \mathrm{~d} \hat{\boldsymbol{K}}
\end{aligned}
$$

for all states $(T, \hat{\boldsymbol{K}})$ and all $0 \leq t_{1}<t<t_{2}$ is considered to be the crucial characterizing feature of the transition probabilities of Markov processes. By multiplication of $p_{1}\left(t_{1}, T_{1}, \hat{\boldsymbol{K}}_{1}\right)$ and integration with respect to $T_{1}$ and $\hat{\boldsymbol{K}}_{1}$, Eq. (10) becomes the following relation between the densities of the one-point distribution and the Markovian transition probability density:

$$
\begin{aligned}
p_{1}\left(t_{2}, T_{2}, \hat{\boldsymbol{K}}_{2}\right)= & \int p_{1}(t, T, \hat{\boldsymbol{K}}) \\
& \cdot p\left(t, T, \hat{\boldsymbol{K}}, t_{2}, T_{2}, \hat{\boldsymbol{K}}_{2}\right) \mathrm{d} T \mathrm{~d} \hat{\boldsymbol{K}} .
\end{aligned}
$$

Provided that within the smallest time intervals, arbitrarily large fluctuations in the turbulence field are improbable, which is the case for our turbulence field, we can derive for $p_{1}$ the following Fokker-Planck equation Eq. (12) (see e.g. Bharucha-Reid 1960, Chap. 3, cf. Arnold 1974, Sect. 2.6), summing over two equal Greek indices $\mu, v=0, \ldots, 3$ in one term. We note that the assumptions made about the existence and continuity of the partial derivatives are fulfilled in our physical system.

$$
\begin{aligned}
\frac{\partial p_{1}}{\partial t}= & -\frac{\partial\left(p_{1} B_{T}\right)}{\partial T}-\frac{\partial\left(p_{1} B_{\hat{\boldsymbol{K}}}^{\mu}\right)}{\partial \hat{K}_{\mu}}+\frac{1}{2} \frac{\partial^{2}\left(p_{1} A_{T}\right)}{\partial T^{2}} \\
& +\frac{1}{2} \frac{\partial^{2}\left(p_{1} A_{\hat{\boldsymbol{K}}}^{\mu v}\right)}{\partial \hat{K}_{\mu} \partial \hat{K}_{v}}+\frac{\partial^{2}\left(p_{1} A_{T \hat{\boldsymbol{K}}}^{\mu}\right)}{\partial \hat{K}_{\mu} \partial T} .
\end{aligned}
$$

Thereby, the coefficient functions for $\mu, v=0, \ldots, 3$ and $\delta>0$ are defined by the following infinitesimal moments, the existence of which is as well presupposed as their independence of the special choice of $\delta$ within the derivation of Eq. (12):

$$
\begin{aligned}
B_{T}(t, T, \hat{\boldsymbol{K}})= & \lim _{\Delta t \rightarrow 0} \frac{1}{\Delta t} \int_{\|(\Delta T, \Delta \hat{\boldsymbol{K}})\|<\delta} \Delta T p(t, T, \hat{\boldsymbol{K}} \\
& t+\Delta t, T+\Delta T, \hat{\boldsymbol{K}}+\Delta \hat{\boldsymbol{K}}) \mathrm{d} \Delta T \mathrm{~d}^{4} \Delta \hat{\boldsymbol{K}}
\end{aligned}
$$

$$
\begin{aligned}
B_{\hat{\boldsymbol{K}}}^{\mu}(t, T, \hat{\boldsymbol{K}})= & \lim _{\Delta t \rightarrow 0} \frac{1}{\Delta t} \underset{\|(\Delta T, \Delta \hat{\boldsymbol{K}})\|<\delta}{\iint_{\mu} \Delta \hat{K}_{\mu} p(t, T, \hat{\boldsymbol{K}},} \\
& t+\Delta t, T+\Delta T, \hat{\boldsymbol{K}}+\Delta \hat{\boldsymbol{K}}) \mathrm{d} \Delta T \mathrm{~d}^{4} \Delta \hat{\boldsymbol{K}},
\end{aligned}
$$

$$
\begin{aligned}
A_{T}(t, T, \hat{\boldsymbol{K}})= & \lim _{\Delta t \rightarrow 0} \frac{1}{\Delta t} \int_{\|(\Delta T, \Delta \hat{\boldsymbol{K}})\|<\delta}(\Delta T)^{2} p(t, T, \hat{\boldsymbol{K}}, \\
& t+\Delta t, T+\Delta T, \hat{\boldsymbol{K}}+\Delta \hat{\boldsymbol{K}}) \mathrm{d} \Delta T \mathrm{~d}^{4} \Delta \hat{\boldsymbol{K}},
\end{aligned}
$$

$$
\begin{aligned}
A_{\hat{\boldsymbol{K}}}^{\mu v}(t, T, \hat{\boldsymbol{K}})= & \lim _{\Delta t \rightarrow 0} \frac{1}{\Delta t} \int_{\|(\Delta T, \Delta \hat{\boldsymbol{K}})\|<\delta} \Delta \hat{\boldsymbol{K}}_{\mu} \Delta \hat{\boldsymbol{K}}_{v} p(t, T, \hat{\boldsymbol{K}}, \\
& t+\Delta t, T+\Delta T, \hat{\boldsymbol{K}}+\Delta \hat{\boldsymbol{K}}) \mathrm{d} \Delta T \mathrm{~d}^{4} \Delta \hat{\boldsymbol{K}}
\end{aligned}
$$

$$
\begin{aligned}
A_{T \hat{\boldsymbol{K}}}^{\mu}(t, T, \hat{\boldsymbol{K}})= & \lim _{\Delta t \rightarrow 0} \frac{1}{\Delta t} \int_{\|(\Delta T, \Delta \hat{\boldsymbol{K}})\|<\delta} \Delta \hat{K}_{\mu} \Delta T p(t, T, \hat{\boldsymbol{K}}, \\
& t+\Delta t, T+\Delta T, \hat{\boldsymbol{K}}+\Delta \hat{\boldsymbol{K}}) \mathrm{d} \Delta T \mathrm{~d}^{4} \Delta \hat{\boldsymbol{K}}
\end{aligned}
$$

In a first step towards a physical derivation of the transition probability density, we assume that the dust formation and growth do not affect the physics of temperature fluctuations. This approximation is justified insofar as the turbulence is due to features 
such as wave fields of the stellar wind and not mainly to the local presence of carbon clusters at the moment of the fluctuation. Because of this stochastic independence, we stipulate, for the stochastic process $\left\{(\Theta, \mathcal{K})_{t} ; t \in \mathbb{R}_{0}^{+}\right\}$as a separation condition, that the transition probability density can be expressed in the form:

$$
\begin{aligned}
p(t, T, \hat{\boldsymbol{K}}, t+\Delta t, T+\Delta T, \hat{\boldsymbol{K}}+\Delta \hat{\boldsymbol{K}})=p_{T}(t, T, t+\Delta t, \\
T+\Delta T) \cdot p_{K}(t, T, \hat{\boldsymbol{K}}, t+\Delta t, \hat{\boldsymbol{K}}+\Delta \hat{\boldsymbol{K}}) .
\end{aligned}
$$

The probability density factor $p_{T}$ is assumed to be independent of $\hat{\boldsymbol{K}}$. $p_{K}$ describes the stochastics of dust formation and its dependence on the fluctuation temperature at the initial time $t$. However, with respect to Eq. (5) and the Markov-presupposition, it is independent of the temperature of the state at time $t+\Delta t$.

\subsection{Temperature fluctuations}

The process component $\Theta(t)$ of the fluctuating temperature deviation can be understood as the result of two factors: (i) if the temperature in a gas volume element varies rapidly due to density fluctuations caused by the collision interaction of particles, a relaxation process will be initiated that results in a state of equilibrium with the environment, assuming that the velocity of the change of $\Theta(t)=T(t)$ in a linear approximation of the temperature behaviour depends only on $T$ with $\dot{\Theta}(0)=0$; (ii) as an additional component including the turbulent temperature fluctuation, we have to assume a stochastically acting fluctuation force, which is supposed to be independent of $\Theta$ insofar as the probabilistic wave field is not considered physically dependent on the actual temperature deviation at the place of dust formation. Both terms together lead to an equation of the Langevin type:

$\mathrm{d} \Theta(t)=-\frac{1}{\lambda} \Theta(t) \mathrm{d} t+\tilde{\sigma} \Gamma(t) \mathrm{d} t$

in which the parameter $\lambda>0$ within the deterministic term determines the order of magnitude of the relaxation time, $\tilde{\sigma}>0$, and the physical description of the temperature fluctuation has to be included in the definition of the stochastic term $\Gamma(t)$. The chosen approach offers a wide range of possibilities for describing temperature turbulences in different concrete physical situations. In the prototype designed here, we assume the following characteristics of the temperature fluctuation on the phenomenological level:

Firstly, there is no reason to assume that $\Gamma$ has a systematic effect on the temperature evolution; the behavior of the mean value $\mathrm{E}(\dot{\Theta}(t))$ is determined by the deterministic term, so that the expectation value equals:

$\mathrm{E}(\Gamma(t))=0$.

Secondly, in a hydrodynamic approximation, simultaneous fluctuations in gas elements that are at significant distances from each other are statistically independent. In terms of time, we have to assume for a gas volume element a multitude of sound and shock waves that are independent of each other and overlap at random; there is then no correlation between two random variables $\Gamma\left(t_{1}\right)$ and $\Gamma\left(t_{2}\right)$ for time spans $\left|t_{2}-t_{1}\right|$ longer than the duration $\Delta \tau$, which is characteristic of an individual stochastic temperature modification, with $\Delta \tau \ll \lambda$. The fluctuation force $\tilde{\sigma} \Gamma(t)$ produces a thermal noise at the place of dust formation by the influence of rapid fluctuations, i.e. a purely random process for which the following expression, using the delta distribution, is valid:

$\operatorname{Cov}\left(\tilde{\sigma} \Gamma\left(t_{1}\right), \tilde{\sigma} \Gamma\left(t_{2}\right)\right)=\tilde{\sigma}^{2} \delta\left(t_{1}-t_{2}\right)$

for all $t_{1}, t_{2} \in \mathbb{R}_{0}^{+}$

Thirdly, at the considered place we assume an overlap of numerous independent influences of random variables. As per the central limit theorem, their total effect is approximately normally distributed. On treating $\Gamma(t)$ formally as stochastic process, we stipulate that the one-point distributions (and consequently all finite-dimensional distributions) follow a Gaussian distribution with expectation value zero.

These requirements can only be fulfilled by a generalized random process, i.e. in general by a continuous linear functional $\Phi(\cdot)$ in the space $\mathcal{D}$ of infinitely differentiable functions $\varphi(\cdot)$ with bounded supports in $\mathbb{R}$, where $\Phi(\varphi)$ is a random variable for each $\varphi$ (see e.g. Gel'fand \& Vilenkin 1964, Chap. III). More precisely, in the stochastic term of Eq. $(15), \Gamma(\varphi)$ has to be defined to be a generalized Gaussian process in which for every choice of linear independent functions $\varphi_{1}, \ldots, \varphi_{n} \in \mathcal{D}$ the random variable $\left(\Phi\left(\varphi_{1}\right), \ldots, \Phi\left(\varphi_{n}\right)\right)$ is normally distributed; as can be verified, the phenomenal specifications are fulfilled by white noise, i.e. for the average and covariance functional of $\Gamma(\varphi)$ we have:

$m(\varphi)=\mathrm{E}(\Gamma(\varphi))=0$

and

$$
\begin{aligned}
\mathrm{C}_{\Gamma}(\varphi, \psi) & =\mathrm{E}((\Gamma(\varphi)-m(\varphi))(\Gamma(\psi)-m(\psi))) \\
& =\int_{-\infty}^{\infty} \varphi(t) \psi(t) \mathrm{d} t
\end{aligned}
$$

for all $\varphi, \psi \in \mathcal{D}$. The white noise, well-defined as a Gaussian process by the average and covariance functional, can be described as a distribution derivative of the generalization $\mathcal{W}(\cdot)$ of the Wiener process $W_{t}$, which, being a homogeneous, stationary Gaussian Markov process, is well-known to be a model of the Brownian motion of a free particle (with $W_{t}=0$ for $t<0$ ):

$$
\mathcal{W}(\varphi)=\int_{-\infty}^{\infty} \varphi(t) W_{t} \mathrm{~d} t \quad(\varphi \in \mathcal{D})
$$

With the usual ordinary, formal, symbolization $\Gamma(t)=d W / d t$, we can now define the special Langevin equation for the fluctuation conditions within our prototype to be:

$\mathrm{d} \Theta(t)=-\frac{1}{\lambda} \Theta(t) \mathrm{d} t+\tilde{\sigma} \mathrm{d} W(t)$

This linear, autonomous stochastic differential equation represents a solution with specified initial value $c$ of the well-known stationary, Gaussian Markovian Ornstein-Uhlenbeck process (cf. e.g. Arnold 1974, Coroll. 8.2.4):

$\Theta_{t}=\mathrm{e}^{-\frac{t}{\lambda}} c+\tilde{\sigma} \int_{0}^{t} \mathrm{e}^{-\frac{t}{\lambda}(t-s)} \mathrm{d} W_{s}$.

As an initial condition, we define $\Theta_{0}$ a normally distributed random variable with expectation value 0 , and by scaling its variance by $\sigma^{2}$, we calculate:

$$
\begin{aligned}
\mathrm{E}\left(\Theta_{t}\right) & =0, \\
\operatorname{Var}\left(\Theta_{t}\right) & =\frac{1}{2} \lambda \tilde{\sigma}^{2}=: \sigma^{2}, \\
\operatorname{Cov}\left(\Theta_{s}, \Theta_{t}\right) & =\sigma^{2} \mathrm{e}^{-\frac{1}{\lambda}|t-s|} .
\end{aligned}
$$


The standard deviation $\sigma>0$, the root mean square temperature fluctuation, indicates the mean turbulent temperature. Because of the correlation function

$$
\begin{aligned}
\rho(s, t) & =\frac{\operatorname{Cov}\left(\Theta_{s}, \Theta_{t}\right)}{\sqrt{\operatorname{Var}\left(\Theta_{s}\right) \operatorname{Var}\left(\Theta_{t}\right)}} \\
& =\mathrm{e}^{-\frac{1}{\lambda}|t-s|}
\end{aligned}
$$

$\lambda$ has to be interpreted as the correlation time characteristic for the turbulence, i.e. the values of the parameter of the fluctuation temperature in the gas element are only correlated within periods of time approximately equal to the time taken to relax to temperature equilibrium. The transition probability density of the Ornstein-Uhlenbeck process can be directly calculated (cf. Todorovic 1992, Propos. 3.7.3). As we know from the theory of diffusion processes, for stochastic differential equations of the type Eq. (20) the searched moments in Eqs. (13a) and (13c) are produced directly from a proposition that holds for the terms of the stochastic differential equation (as drift vector and diffusion matrix, see Arnold 1974, Theorem 9.3.1):

$\lim _{\Delta t \rightarrow 0} \frac{1}{\Delta t} \int \Delta T p_{\Theta}(t, T, t+\Delta t, T+\Delta T) \mathrm{d} \Delta T=-\frac{1}{\lambda} T$,

and

$$
\lim _{\Delta t \rightarrow 0} \frac{1}{\Delta t} \int(\Delta T)^{2} p_{\Theta}(t, T, t+\Delta t, T+\Delta T) \mathrm{d} \Delta T=2 \frac{\sigma^{2}}{\lambda} .
$$

The transition probability density of a stochastic process which is a solution of a stochastic differential equation of the general type, to which Eq. (20) belongs, produces a fundamental solution of a partial differential equation of the Kolmogorov forward (or Fokker-Planck) type, which can be directly formulated by use of the drift vector and the diffusion matrix (see Arnold 1974, Sect. 9.4).

\subsection{Process component of dust formation}

For the determination of the transition probability density $p_{K}$ in Eq. (14), the following points must be considered.

Firstly, the stochastic nature of dust formation is due entirly to its dependence on the temperature fluctuation. Secondly, the solution $\boldsymbol{K}_{t}\left(t_{1}, \boldsymbol{K}^{1}\right)$ of Eq. (6) for the initial value $\boldsymbol{K}_{t_{1}}=\boldsymbol{K}^{1}$ transports the state $\boldsymbol{K}^{1}$ at time $t_{1}$ to the state $\boldsymbol{K}_{t}$ at time $t$ within the time period $t-t_{1}$.

With a canonical choice of random variables (which is always possible as a corollary of the fundamental theorem of Kolmogorov), $\mathcal{K}_{t}(\hat{\boldsymbol{K}})=\hat{\boldsymbol{K}}_{t}$ for all $t$, the value of the random variables can, for small time steps $\Delta t=t-t_{1}$, be set equal to the value

$\hat{\boldsymbol{K}}_{t}=\left.\boldsymbol{K}_{t}\left(t_{1}, \boldsymbol{K}^{1}\right)\right|_{T_{1}}$

rendered by the deterministic calculation for a given temperature deviation $T_{1}$ at the initial time $t_{1}$.

The probability $P_{\mathcal{K}}$ of the transition from the state of process $\hat{\boldsymbol{K}}^{1}$ at time $t_{1}$ to a state within the value set $B$ at time $t$ for sufficiently small time periods is therefore

$$
\begin{aligned}
P_{\mathcal{K}}\left(t_{1}, T_{1}, \hat{\boldsymbol{K}}^{1}, t, B\right) & =\chi_{\boldsymbol{K}_{t}\left(t_{1}, \boldsymbol{K}^{1}\right)}(B) \\
& =\left\{\begin{array}{l}
1, \text { for }\left.\boldsymbol{K}_{t}\left(t_{1}, \boldsymbol{K}^{1}\right)\right|_{T_{1}} \in B \\
0, \text { for }\left.\boldsymbol{K}_{t}\left(t_{1}, \boldsymbol{K}^{1}\right)\right|_{T_{1}} \notin B .
\end{array}\right.
\end{aligned}
$$

As a consequence of the first order of the differential equations of dust formation Eq. (6), this is a Markovian transition probability: For the approximation

$\Delta \boldsymbol{K}=(\mathrm{A} \cdot \boldsymbol{K}+\boldsymbol{b}) \Delta t$

at small $\Delta t>0$ and using the $\delta$-distribution, we obtain for the transition probability density:

$$
p_{K}(t, T, \hat{\boldsymbol{K}}, t+\Delta t, \hat{\boldsymbol{K}}+\Delta \hat{\boldsymbol{K}})=\delta\left(\Delta \hat{\boldsymbol{K}}-(\mathrm{A} \cdot \hat{\boldsymbol{K}}+\boldsymbol{b})_{T} \Delta t\right)
$$

to which Eq. (10) obviously applies.

In a conceptual respect, the analytical dependence of the dust formation moments according to Eq. (6) has now been transferred into a stochastic dependence of the random variables $\left(\hat{K}_{\mu}\right)_{t}$ described by the transition probability density Eq. (29).

To specify the coefficient functions (Eq. (13)) within the Fokker-Planck equation Eq. (12), we calculate (and formulate by use of the summation convention):

$$
\begin{aligned}
\int \Delta \hat{K}_{\mu} p_{K} \mathrm{~d}^{4} \Delta \hat{\boldsymbol{K}} & =\sum_{v=0}^{3}\left(A_{\mu v} \hat{K}_{v}+b_{\mu}\right) \Delta t \\
& =\left(A_{\mu \nu} \hat{K}_{v}+b_{\mu}\right) \Delta t,
\end{aligned}
$$

and

$\int \Delta \hat{K}_{\mu} \Delta \hat{K}_{v} p_{K} \mathrm{~d}^{4} \Delta \hat{\boldsymbol{K}}=\left(A_{\mu \lambda} \hat{K}_{\lambda}+b_{\mu}\right)\left(A_{v \rho} \hat{K}_{\rho}+b_{v}\right)(\Delta t)^{2}$

for all $\mu, v=0, \ldots, 3$.

\subsection{Set of Fokker-Planck equations}

The Markovian property of $p_{T}$ and $p_{K}$ is carried forward to the entire process; the assumptions made in the derivation of the Fokker-Planck equation Eq. (12) are fulfilled, in particular the regularity assumptions for the partial derivative in Eq. (12), the existence of the moments Eq. (13) (even uncut), and the improbability of arbitrarily large fluctuations in the smallest time intervals: for any $\delta>0$, we calculate:

$$
\begin{array}{r}
\lim _{\Delta t \rightarrow 0} \frac{1}{\Delta t} \iint_{\|(\Delta T, \Delta \hat{\boldsymbol{K}})\|>\delta} p(t, T, \hat{\boldsymbol{K}}, t+\Delta t, T+\Delta T, \\
\hat{\boldsymbol{K}}+\Delta \hat{\boldsymbol{K}}) \mathrm{d} \Delta T \mathrm{~d}^{4} \Delta \hat{\boldsymbol{K}}=0 .
\end{array}
$$

With a transition probability density $p=p_{T} p_{K}$ of the stochastic process $(\Theta, \mathcal{K})_{t}$ determined in this way, we calculate for Eqs. (24), (25), (30), and (31), for the coefficient functions in Eq. (13) of the Fokker-Planck equation:

$$
\begin{array}{ll}
B_{T}=-\frac{1}{\lambda} T, & B_{\hat{\boldsymbol{K}}}^{\mu}=A_{\mu v} \hat{K}_{v}+b_{\mu}, \\
A_{T}=2 \frac{\sigma^{2}}{\lambda}, & A_{\hat{\boldsymbol{K}}}^{\mu \nu}=A_{T \hat{\boldsymbol{K}}}=0
\end{array}
$$

for $\mu, v=0, \ldots, 3$. For the one-point probability density $p_{1}(t, T, \hat{\boldsymbol{K}})$ the Fokker-Planck equation is then:

$$
\begin{aligned}
\frac{\partial p_{1}}{\partial t}=\frac{1}{\lambda}\left(\frac{\partial}{\partial T} T+\sigma^{2}\right. & \left.\frac{\partial^{2}}{\partial T^{2}}\right) p_{1} \\
& -\frac{\partial}{\partial \hat{K}_{\mu}}\left(p_{1}\left(A_{\mu \nu} \hat{K}_{v}+b_{\mu}\right)\right) .
\end{aligned}
$$


In the situation of physical observation, we average over the statistical ensemble of gas elements, which means that in the theoretical approach the expectation values play a decisive role. With respect to the random variable $\hat{K}_{\rho}$ we therefore define the first moment to be:

$Q_{\rho}=\int \hat{K}_{\rho} p_{1} \mathrm{~d}^{4} \hat{\boldsymbol{K}} \quad(\rho=0, \ldots, 3)$.

Applying the operator $\int \mathrm{d}^{4} \hat{\boldsymbol{K}} \hat{K}_{\rho}$ to equation Eq. (34), we obtain:

$$
\begin{aligned}
\frac{\partial Q_{\rho}}{\partial t}=\frac{1}{\lambda} \frac{\partial}{\partial T}\left(T Q_{\rho}\right) & +\frac{\sigma^{2}}{\lambda} \frac{\partial^{2} Q_{\rho}}{\partial T^{2}} \\
& -\int \frac{\partial}{\partial \hat{K}_{\mu}}\left(p_{1}\left(A_{\mu \nu} \hat{K}_{v}+b_{\mu}\right)\right) \hat{K}_{\rho} \mathrm{d}^{4} \hat{\boldsymbol{K}} .
\end{aligned}
$$

For the final term on the right hand side, we calculate:

$$
\begin{aligned}
& \int \frac{\partial}{\partial \hat{K}_{\mu}}\left(p_{1}\left(A_{\mu \nu} \hat{K}_{v}+b_{\mu}\right)\right) \hat{K}_{\rho} \mathrm{d}^{4} \hat{\boldsymbol{K}}= \\
& \int \frac{\partial}{\partial \hat{K}_{\mu}}\left(p_{1}\left(A_{\mu \nu} \hat{K}_{v}+b_{\mu}\right) \hat{K}_{\rho}\right) \mathrm{d}^{4} \hat{\boldsymbol{K}} \\
& -\int p_{1}\left(A_{\mu \nu} \hat{K}_{v}+b_{\mu}\right) \frac{\partial}{\partial \hat{K}_{\mu}} \hat{K}_{\rho} \mathrm{d}^{4} \hat{\boldsymbol{K}} .
\end{aligned}
$$

After the application of the theorem of Gauss, the first summand on the right hand side becomes zero because of the probability normalization on 1 over the entire state space (and the square integrability of $\hat{K}_{\rho}$ ). For the second summand and its random variables, we receive:

$$
\begin{aligned}
\int p_{1}\left(A_{\mu v} \hat{K}_{v}+b_{\mu}\right) \frac{\partial}{\partial \hat{K}_{\mu}} & \hat{K}_{\rho} \mathrm{d}^{4} \hat{\boldsymbol{K}} \\
& =A_{\rho v} Q_{v}+b_{\rho} \int p_{1} \mathrm{~d}^{4} \hat{\boldsymbol{K}} \\
& =A_{\rho v} Q_{v}+b_{\rho} p_{1}^{T}
\end{aligned}
$$

where $p_{1}^{T}$ is the temperature distribution density remaining after the integration over the entire $\hat{\boldsymbol{K}}$-state space, which is in this case given by the Gaussian density of the Ornstein-Uhlenbeck process (with respect to the system Eq. (22)):

$p_{1}^{T}(t, T)=\left(2 \pi \sigma^{2}\right)^{-\frac{1}{2}} \mathrm{e}^{-\frac{T^{2}}{2 \sigma^{2}}}$.

The Fokker-Planck equation system for the one-point probability density $p_{1}(t, T, \hat{\boldsymbol{K}})$ for $\rho=0, \ldots, 3$ is then given by the following coupled system of four second order linear partial differential equations of the parabolic type:

$\frac{\partial Q_{\rho}}{\partial t}=\frac{1}{\lambda} \frac{\partial}{\partial T}\left(T Q_{\rho}\right)+\frac{\sigma^{2}}{\lambda} \frac{\partial^{2} Q_{\rho}}{\partial T^{2}}+A_{\rho v} Q_{v}+b_{\rho} p_{1}^{T}$.

The solutions provide the sought after expectation values for the random variables $\hat{K}_{0}, \ldots, \hat{K}_{3}$, which constrain the dust formation in a probabilistic way:

$$
\begin{aligned}
\mathrm{E}\left(\hat{K}_{\rho}\right)(t) & =\iint \hat{K}_{\rho} p_{1}(t, T, \hat{\boldsymbol{K}}) \mathrm{d}^{4} \hat{\boldsymbol{K}} \mathrm{d} T \\
& =\int Q_{\rho}(t, T) \mathrm{d} T \quad(\rho=0, \ldots, 3) .
\end{aligned}
$$

For the numerical treatment, we define the corresponding conditional moments:

$q_{\rho}(t, T)=\frac{Q_{\rho}(t, T)}{p_{1}^{T}(t, T)}$.

With respect to Eq. (39), we obtain for Eq. (40) after division by $p_{1}^{T}>0$ :

$\frac{\partial q_{\rho}}{\partial t}=-\frac{1}{\lambda} T \frac{\partial q_{\rho}}{\partial T}+\frac{\sigma^{2}}{\lambda} \frac{\partial^{2} q_{\rho}}{\partial T^{2}}+A_{\rho v} q_{v}+b_{\rho}$

for $\rho=0, \ldots, 3$.

\section{Gas-box models}

Since the local radiative acceleration is a function of the degree of condensation $f_{\mathrm{c}}\left(\sim q_{3}\right)$, the direct application of the equation system Eq. (43) to a wind description yields the problem that different values of $T$ would produce different accelerations for different temperature deviations. The important feedback of the dust formation onto the underlying wind structure can therefore not be described using Eq. (43). If, however, the correlation length $\lambda$ of the fluctuations is so small, that the process occurs at the microturbulent limit, this problem can be evaded because the values of $q_{\rho}$ are then almost equal for all values of $T$. To determine i) for which values of $\lambda$, the stochastic dust formation can be described in the microturbulent limit case; and ii) for which values of $\sigma$, a significant influence of fluctuations on the dust formation can be expected, we applied the formalism described in Sect. 3 to a set of gas-box models, which present typical trajectories of volume elements in a stationary AGB wind. For the reasons stated above, no feedback of the dust formation on the hydrodynamical structure of the model is included. We assumed a constant wind velocity of $20 \mathrm{~km} \mathrm{~s}^{-1}$, which corresponds to a typical end velocity of an AGB wind. This assumption is required because the solution of the Fokker-Plank system Eq. (43), based on a more realistic wind model of velocity $v(r)$ that increases with distance $r$, produces almost complete condensation in the slow part of the wind; this is due to the lack of consideration of the effect of radiative acceleration on the newly formed grains, which then causes them to become unrealistically long in the region of efficient dust condensation. We adopted a high carbon-to-oxygen ratio, which, in turn, facilitates the dust formation process in the artificial situation. In these prototypic calculations, we aim to constrain the parameter space of $\sigma$ and $\lambda$, and estimate the influence of the temperature fluctuations on the dust formation under AGB conditions; this would enable us to implement consistently the effects of the temperature fluctuations on dynamical model calculations as presented in Sect. 5.

The gas-box wind model (see box) is used as input to the Fokker-Planck system Eq. (43). We assume that at a given moment in time $t_{1}$, the densities $\rho\left(t_{1}, T\right)$ are coupled adiabatically along the $T$-axis.

These gas box models can be interpreted most effectively as the temporal development of the dust component in a volume 
comoving with the given wind:

$$
\begin{aligned}
\bar{T} & =T_{\star} \sqrt{\frac{R_{\star}}{r}} \\
R_{\star} & =3.7 \times 10^{13} \mathrm{~cm} \\
T_{\star} & =2500 \mathrm{~K} \\
v_{\text {wind }} & =20 \mathrm{~km} \mathrm{~s}^{-1} \\
M_{\star} & =1 M_{\odot} \\
\dot{M} & =2 \times 10^{-5} M_{\odot} \mathrm{a}^{-1} \\
\bar{\rho}(r) & =\frac{\dot{M}}{4 \pi r^{2} v_{\text {wind }}} \\
\epsilon_{\mathrm{C}}: \epsilon_{\mathrm{O}} & =3
\end{aligned}
$$

We start the integration at $r=R_{\star}$ and $t=0$ in a dust-free situation. The Fokker-Planck system Eq. (43) is then integrated forward in time with the Cranck-Nicolson method. Using $t=$ $r / v_{\text {wind }}$, the functions $\bar{T}(t)$ and $\bar{\rho}(t)$ are used as input for the transition probabilities. By discretization with a second-order difference scheme, the Fokker-Planck system finds its numerical analogon in a set of linear systems of equations, which can be represented by a tridiagonal matrix and then solved by a standard solver. Along the axis of the temperature fluctuation ( $T$-axis), we truncated the integration domain at $T= \pm \gamma \sigma$, with $\gamma \approx 8$; an integration from $-\infty$ to $+\infty$ is numerically impossible and physically meaningless. The integration domain must be selected to be sufficiently large to ensure that the boundary conditions have no influence on those parts of the domain that contribute significantly to the Gaussian-weighted averaged expectation values. As a boundary condition, we used the corresponding deterministic path, and note that other non-pathological choices produced the same results.

In most calculations (Sects. 4.1-4.3), we assumed the dust temperature $T_{\mathrm{d}}$ to equal the gas temperature $T_{\mathrm{g}}$. This corresponds to assuming an effective energy exchange between gas and dust, e.g. slow fluctuations (large $\lambda$ ), high densities (i.e. high collision rates). To estimate the effects of dust temperature deviating from gas temperature, we performed a set of calculations assuming $T_{\mathrm{d}}=\bar{T}$, which could be interpreted as rapid fluctuations in a thin gas (see Sect. 4.4).

We solved the equation system Eq. (43) on top of the gas-box wind model described above. In Sects. 4.1 and 4.2, we present two parameter studies for $\sigma$ and $\lambda$. In Sect. 4.3, we discuss the behaviour of the solution compared with a set of purely deterministic calculations for the same wind model. In Sect. 4.4, we present some models, where we assumed the dust temperature to remain at the mean temperature with the gas temperature continuing to fluctuate.

\subsection{Influence of the mean turbulent temperature}

The results of a study of different values of $\sigma$, i.e. root mean square turbulent temperatures, are shown in Fig. 1. In the top panel, the mean values of the radial temperature and density structure are given; these values are used as input of the calculation (see box in the head of this section). In Panels 2 and 3, the mean nucleation rate $\left\langle J_{N_{\ell}}\right\rangle$ and the mean net growth rate $\left\langle\tau^{-1}\right\rangle$ are plotted, which correspond to an integration of $J_{N_{\ell}}(T, \rho)$ and

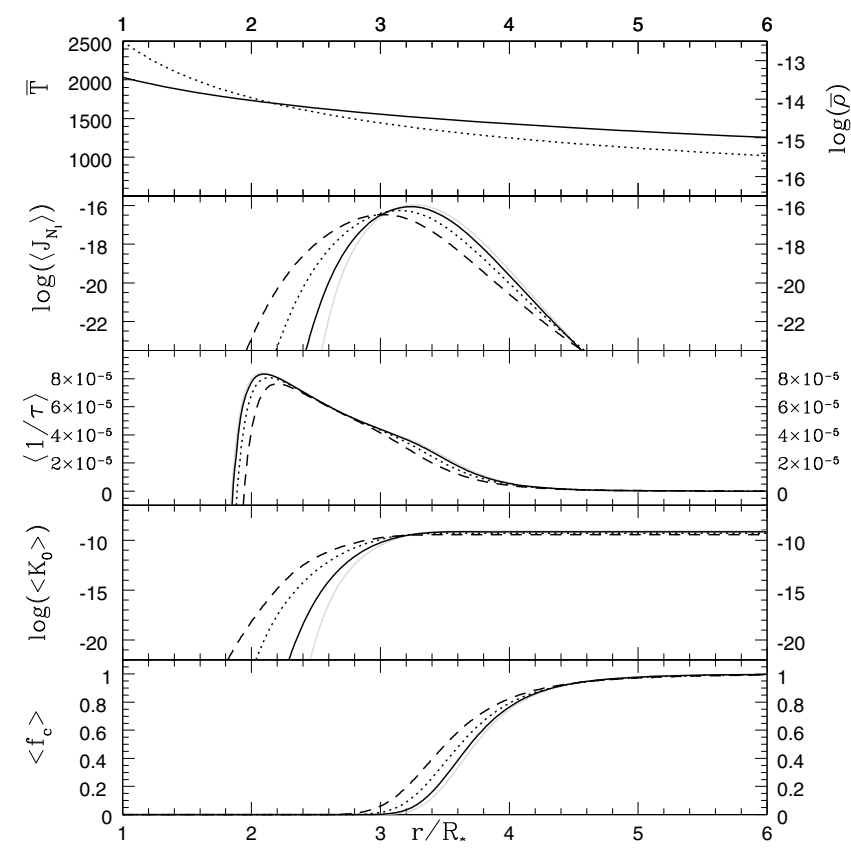

Fig. 1. Parameter study for various mean turbulent temperatures $\sigma$. Top panel: mean temperature (dotted) and mean density (logarithmic, solid). Panel 2: average nucleation rate (black, full: $20 \mathrm{~K}$, dotted: $40 \mathrm{~K}$, dashed 60 K, grey: deterministic model). Panel 3: average net growth rate. (The deterministic curve is covered by the black curve for $\sigma=20 \mathrm{~K}$.) Panel 4: expectation value of the number density of dust grains (logarithmic). Panel 5: expectation value of the degree of condensation. $\lambda=10^{4} \mathrm{~s}$ for all plots.

$\tau^{-1}(T, \rho)$ over all $T$-paths weighted by the probability function $p_{1}^{T}(T)$ (see Eq. (39)):

$$
\begin{aligned}
& \left\langle J_{N_{\ell}}\right\rangle=\int_{-T_{\max }}^{+T_{\max }} p_{1}^{T}(T) J(T, \rho) \mathrm{d} T, \\
& \left\langle\tau^{-1}\right\rangle=\int_{-T_{\max }}^{+T_{\max }} p_{1}^{T}(T) \tau^{-1}(T, \rho) \mathrm{d} T,
\end{aligned}
$$

where $J_{N_{\ell}}(T, \rho)$ and $\tau^{-1}(T, \rho)$ are evaluated at the gas temperature

$T_{\mathrm{g}}=\bar{T}+T$,

and the density

$\rho=\left(\frac{\bar{T}+T}{\bar{T}}\right)^{\frac{1}{\kappa-1}} \bar{\rho}$, with

$\kappa=\frac{f+2}{f}$,

and $f$ being the number of degrees of freedom of the gas. In this work, we chose $f=3$, which corresponds to an atomic ideal gas. We therefore assume the density to couple adiabatically to the temperature fluctuation. Panel 4 shows the expectation value of the number density of dust particles

$E\left(n_{\mathrm{d}}\right)=\left\langle n_{\mathrm{d}}\right\rangle=\int_{-T_{\max }}^{+T_{\max }} q_{0}(T) p_{1}^{T}(T) \mathrm{d} T$, 
and Panel 5 provides the expectation value of the degree of condensation

$E\left(f_{\mathrm{c}}\right)=\left\langle f_{\mathrm{c}}\right\rangle=\frac{1}{\epsilon_{\mathrm{C}}} \int_{-T_{\max }}^{+T_{\max }} q_{3}(T) p_{1}^{T}(T) \mathrm{d} T$.

We note that the thermodynamical structure is an input to the calculation, whereas $E\left(n_{\mathrm{d}}\right)$ and $E\left(f_{\mathrm{c}}\right)$ are calculated from the solutions $q_{\rho}$ of the Fokker-Planck system Eq. (43). The functions $J$ and $\tau^{-1}$ are calculated at every time step using the given thermodynamical structure as input and the $q_{3}$-solution of the FokkerPlanck system, which couples back into the equations by means of the depleted carbon concentration $\epsilon_{\mathrm{C}}(t, T)=\epsilon_{\mathrm{C}, 0}-q_{3}(t, T)$.

For small rms-temperature fluctuations, the models approach the behaviour of the deterministic calculation, as can be seen in Panels 2-5. This is the expected behaviour because using for example the sequence

$\delta_{n}(\cdot)=n \mathrm{e}^{-\pi n^{2}(\cdot)^{2}}$

to represent the $\delta$-distribution, it can be seen that the probability distribution of the Ornstein-Uhlenbeck process (cf. Eq. (39)) converges towards the $\delta$-distribution for small widths $\sigma$ :

$p_{1}^{T}=\left(2 \pi \sigma^{2}\right)^{-\frac{1}{2}} \mathrm{e}^{-\frac{T^{2}}{2 \sigma^{2}}} \rightarrow \delta(T) \quad$ for $\quad \sigma \rightarrow 0$.

An analytical calculation therefore produces the result that the expectation values of the random variables solve the deterministic equation system Eq. (6): this results from Eqs. (41), (42), and (43) with

$\mathrm{E}\left(\hat{K}_{\rho}\right)=q_{\rho}(t, 0)$

and

$\int \frac{\partial}{\partial T} T Q_{\rho} \mathrm{d} T=0$

by integration over the $T$-space:

$\frac{\partial}{\partial t} \mathrm{E}\left(\hat{K}_{\rho}\right)=A_{\rho v}(\bar{T}(t)) \mathrm{E}\left(\hat{K}_{v}\right)+b_{\rho}(\bar{T}(t))$

for all $\rho, v=0, \cdots, 3$.

The most important physical effect of the fluctuations is the earlier onset of nucleation (panel 2), which becomes more pronounced with increasing $\sigma$. The earlier onset of the nucleation is a direct consequence of the lower temperature in the cool paths, which facilitates nucleation. In the cool paths seeds are therefore produced, when $S>S_{\text {crit }}$, resulting in non-zero values for the $q_{\rho}$, which persist after fluctuations, because these grains do not re-evaporate until $S$ drops below 1 (see also Fig. 3). The early nucleation is also obvious in Panel 4: high number densities of dust grains are reached about $0.5 R_{*}$ deeper inside the wind, for $\sigma=60 \mathrm{~K}$ than for $\sigma=20 \mathrm{~K}$. The final particle size, however, will decrease with the enhanced nucleation rate $J$, because the condensable material is condensed into more individual grains and further condensation is stopped by depletion of the gas phase.

Panel 5 shows that the region of runaway condensation via growth of the existing grains is shifted about $0.25 R_{*}$ inward for $\sigma=60 \mathrm{~K}$ with respect to $\sigma=20 \mathrm{~K}$.

The slightly lower growth rate for $\sigma=60 \mathrm{~K}$ with respect to that of the $\sigma=20 \mathrm{~K}$-model, can be explained by the earlier depletion, due to the larger amount of condensed material.

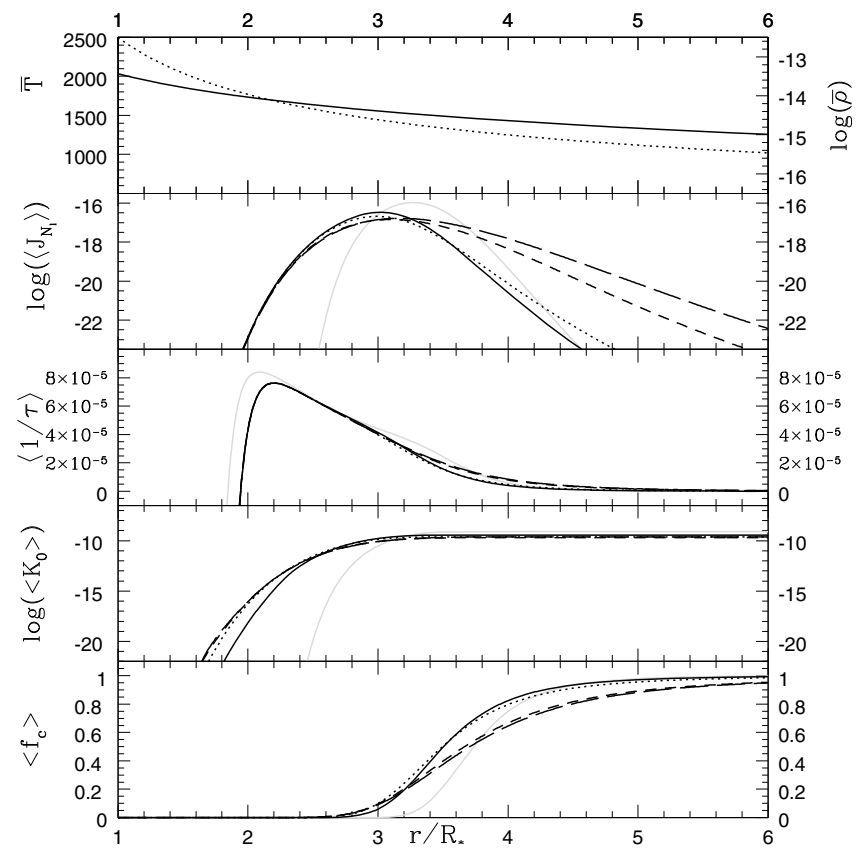

Fig. 2. Parameter study for various correlation times. The depicted quantities are identical to Fig. 1. Grey: deterministic; black full: $\lambda=$ $10^{4} \mathrm{~s}$; dotted: $10^{7} \mathrm{~s}$; dashed: $10^{8} \mathrm{~s}$; long dashed: $10^{9} \mathrm{s.} \sigma=60 \mathrm{~K}$ for all plots.

\subsection{Influence of the correlation times}

The results of a study of various values of the correlation time $\lambda$ of fluctuations with a rms-temperature fluctuation $\sigma=60 \mathrm{~K}$ are shown in Fig. 2.

In general, the dependence of the model calculations on the correlation time is rather small. We observe a slower decline in the nucleation rate for long correlation times (panel 2), alltough the overall impact of this effect on the dust moments (panels 4 and 5) is small.

We can identify two extreme cases, (i) the microturbulent limit $(\lambda \rightarrow 0)$; and (ii) the macroturbulent limit case $(\lambda \rightarrow \infty)$. Mathematically, it is the operator

$\frac{1}{\lambda}\left(\frac{\partial}{\partial T} T+\sigma^{2} \frac{\partial}{\partial T^{2}}\right)$

in the Fokker-Planck system Eq. (40) that yields the influence of the correlation time $\lambda$. Since $q_{\rho}(t, T)$ represents the part of dust formation for which the temperature deviation is in the interval $[T, T+\mathrm{d} T)$, the Fokker-Planck operator describes the interaction between these different modes of the turbulence (cf. e.g. Traving 1975, Sect. 4).

In a microturbulent situation, the influence of the operator, and with it, the influence of the fluctuation, become overwhelming. Physically, this implies that the fluctuations are rapid compared with the processes controlling the dust physics and are therefore uncorrelated on the (longer) timescales governing the condensation process. The different temperature paths are then strongly coupled (cf. Eq. (23)). In this case, it should be possible to find an easier way of calculating the influence of the temperature fluctuations, than the method presented here, because the $\lambda$-dependence vanishes. It can indeed be seen in Fig. 2 that the expectation values of the degree of condensation $f_{\mathrm{c}}$ (panel 5) are nearly identical for $\lambda=10^{4} \mathrm{~s}$ and $\lambda=10^{7} \mathrm{~s}$.

The $f_{\mathrm{c}}$-plots for $\lambda=10^{8} \mathrm{~s}$ and $\lambda=10^{9} \mathrm{~s}$ in Fig. 2 are also almost identical. They represent the macroturbulent limit case 


\section{stochastic fC}

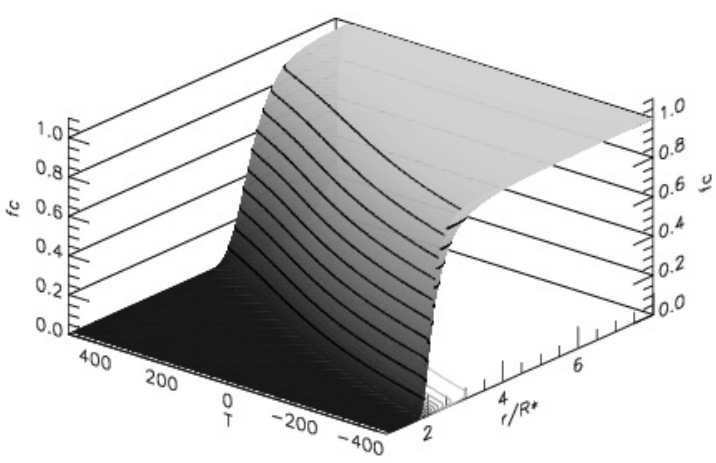

stochastic $\log (q 0)$

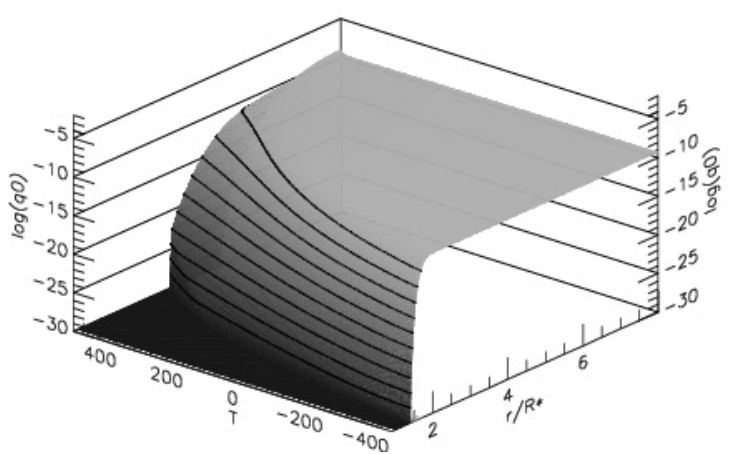

deterministic fc
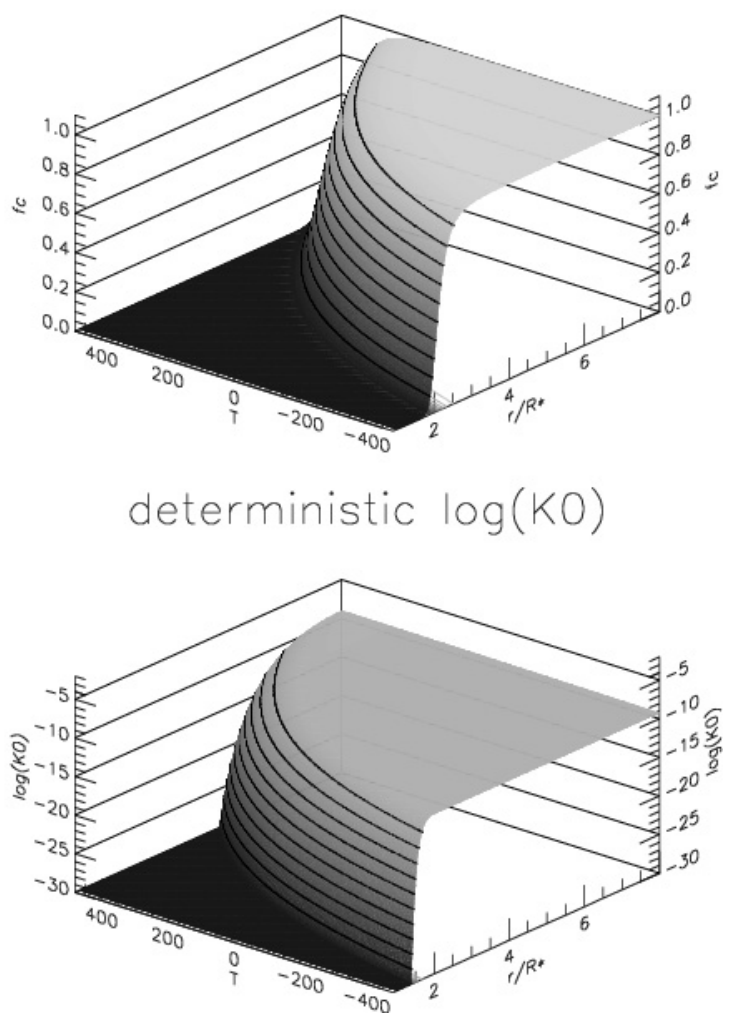

Fig. 3. Degree of condensation $f_{\mathrm{c}}$ (top) and number density of dust grains (bottom) along the $T-r$-plane in the deterministic (right) and the stochastic (left) case. $T$ is the temperature deviation from the mean temperature (see Fig. 1 top panel). $K_{0}$ and $q_{0}$ are again normated to the total number density of hydrogen cores $n_{\hat{\mathrm{H}}} . q_{0}$ (bottom left) results from the first moment Eq. (35) of $\hat{K}_{0}$ via Eq. (42). The $K_{0}$ on the right are the result of a set of independent deterministic calculations along the single paths $(\bar{T}+T)$, i.e. there is no coupling along the $T$-axis in the deterministic case (right). The degree of condensation $f_{\mathrm{c}}=q_{3} / \epsilon_{\mathrm{C}, 0}$ or $K_{3} / \epsilon_{\mathrm{C}, 0}$ must be understood accordingly, $\left(\sigma=60 \mathrm{~K}, \lambda=10^{8} \mathrm{~s}\right)$.

for which the correlation time of the fluctuation is very long compared with the timescales governing the condensation process, i.e. the interaction between the different temperature paths is weak. Due to the slow relaxation, the gas volume element remains at the offset temperature in the case of temperature disturbance. The separation of modes causes a quasi-deterministic behaviour of the expectation values. In the mathematical macroturbulent limit, the operator (53) vanishes and the Fokker-Planck system Eq. (43) for $q_{\rho}$ corresponds to the deterministic system Eq. (6) for $K_{\rho}$. The expectation values of $q_{\rho}$ then become the weighted mean of the deterministic moments $K_{\rho}$, integrated over all temperature paths:

$E\left(q_{\rho}\right)=\int p_{1}^{T}(T) K_{\rho}(T) \mathrm{d} T$

In general, we note that the models appear to switch between the micro- and the macroturbulent limit cases, i.e. the mesoturbulent case occurs only for a very narrow range of $\lambda$-values.

\subsection{Dust physics under the influence of the fluctuations}

Figure 3 shows a comparison between the stochastic calculation (solution of the Fokker-Planck system Eq. (43), left hand side) and a set of deterministic calculations (solution of Eq. (6), right hand side) on the same $T_{\mathrm{g}}(t, T) / \rho(t, T)$-structure. In contrast to the solutions $q_{\rho}$ of the Fokker-Planck system, we note that the deterministic plot on the right side represents a set of isolated calculations of the $K_{\rho}$ for each path of constant temperature deviation $T$, i.e. the $K_{\rho}$ do not "interact" along the $T$-axis.

Due to the choice of boundary conditions, the boundary paths $(T= \pm \gamma \sigma)$ of the stochastic system are identical to the corresponding deterministic paths. However, Figs. 3 and 4 show clearly that the influence of the boundaries on the solutions $q_{\rho}$ along the $(t, T)$-plane is weak; we note in particular the rapidly diminishing influence of the high temperature boundary visible in the lower left panel of Fig. 4. The influence of the boundaries on the expectation values is even orders of magnitudes smaller, because the $q_{\rho}$ are weighted by the Gaussian-bell-shaped probability function $p_{T}$, (see Eqs. (46), (47), and (39)).

Figure 4 shows the net growth rate (top) and nucleation rate (bottom) in the stochastic (left) and deterministic cases (right). Both plots look very similar, because the only coupling mechanism along the $T$-axis (in the stochastic case) is the modified carbon abundance, which results from the modified degrees of condensation via the stochastic transport of $q_{\rho}$ by the first two terms on the right side of Eq. (43). The similarity of the growth rate (upper plots in Fig. 4) is the consequence of the fact that the depicted growth rate is the hypothetical rate at which a grain would grow, if it were present. This rate usually peaks before efficient nucleation begins (see Fig. 1), and the peak in the growth rate in Fig. 4 is therefore hardly influenced by the stochastical fluctuations, even though it dominates the plot. For the same reason, the stochastic nucleation rates (bottom left in Fig. 4) are not modified in the part before (or inside) of their peak. Only after efficient nucleation occurs at least somewhere in the integration 


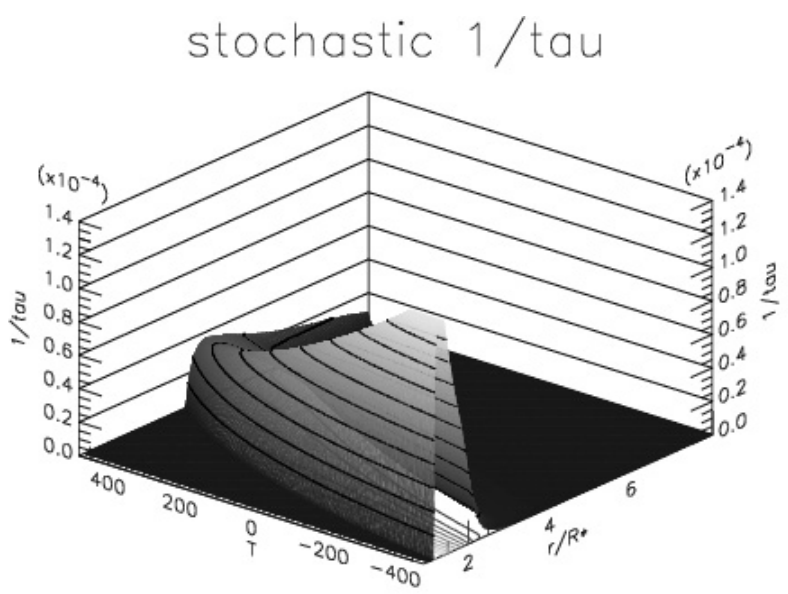

stochastic $\log (J)$

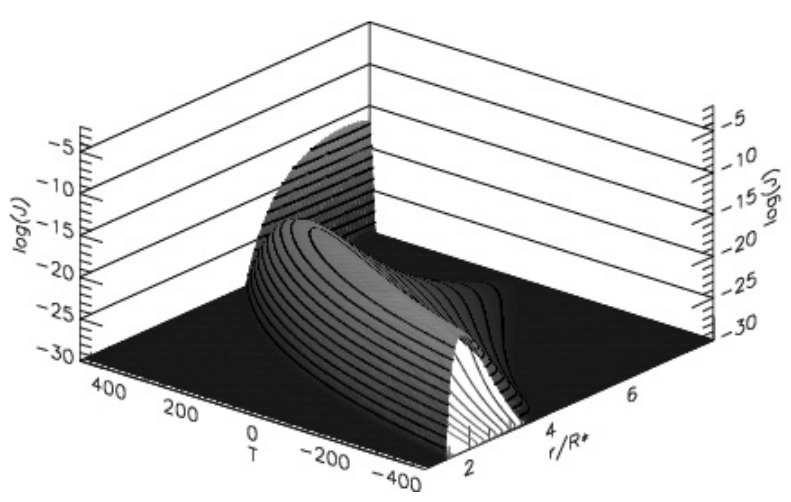

deterministic $1 /$ tau

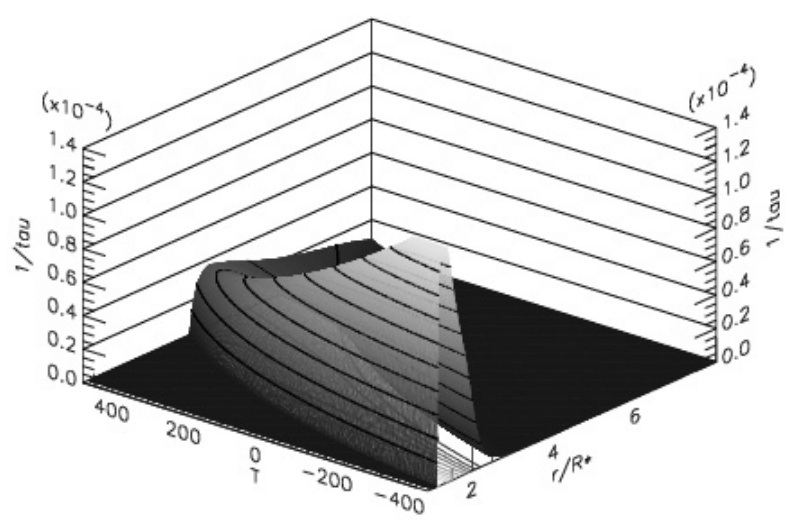

deterministic $\log (\mathrm{J})$

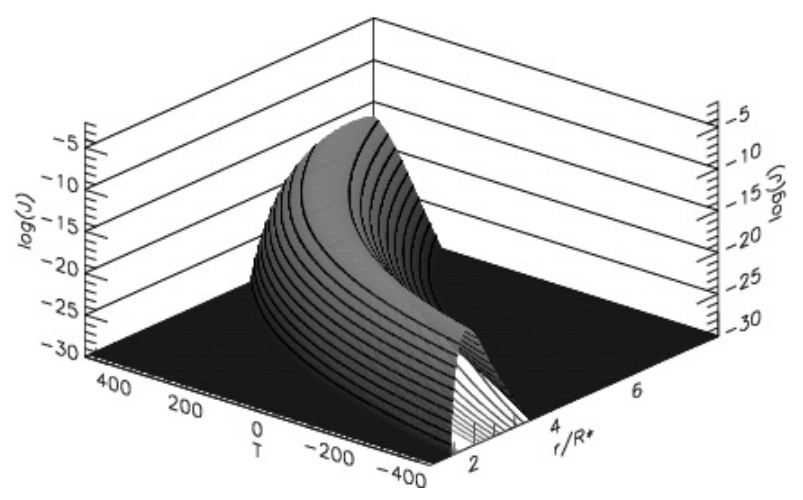

Fig. 4. Net growth rate (top) and nucleation rate (bottom) for deterministic (right) and stochastical calculations respectively. The stochastic plots on the left are coupled by the chemical consumption of monomers, i.e. by the $q_{3}$-solution of the Fokker-Planck system Eq. (43) on the $T-r$-plane, whereas on the right - we again plot a sample of deterministic calculations. $\left(\sigma=60 \mathrm{~K}, \lambda=10^{8} \mathrm{~s}\right.$.

domain, can the coupling mechanism in terms of $q_{\rho}$ be established; for values of $r \gtrsim 2.5 R_{\star}$ (i.e. behind the peak in the nucleation rate, for $T<0$ ), the difference between the stochastic and deterministic cases (bottom right) can indeed be seen.

We now consider more carefully the influence of the temperature fluctuations. The location at which the net growth rate ${ }^{4}$ becomes positive is always closer to the star than the location at which nucleation from the gas phase initiates. This is easily understood because (hypothetical) dust grains should grow for $S \gtrsim 1$, whereas the nucleation of new grains from the gas phase requires a supersaturation $S>S_{\text {crit }}>1$.

If fluctuations are present, i.e. if the different $T$-paths are coupled by the Fokker-Planck system Eq. (43), the grains that nucleate in the cooler paths are mixed into the hotter paths, where they will grow as long as $S>1$. The fluctuations therefore generate a mechanism that retains nuclei in a thermodynamic domain, where, in a deterministic situation, nucleation would not occur, due to a lack of supersaturation. This effect is clearly visible in Fig. 3: both, the number density of dust particles $q_{0}$ and the degree of condensation $f_{\mathrm{c}}$ begin to rise earlier and reach their final level earlier in each path of the stochastic calculation (left) than in the corresponding path of the deterministic calculation (right).

\footnotetext{
${ }^{4}$ Note that the net growth rate is the hypothetical rate at which, if present, a cluster would grow or evaporate. This rate can be positive even if no dust at all is present.
}

\subsection{Models without coupling between dust and gas temperature}

The nucleation and growth rates depend sensitively on differences between gas and dust temperature, because in statistical equilibrium the creation rate increases with the gas temperature, while the corresponding evaporation rate increases with the temperature of the grain (see Patzer et al. 1998). Figure 5 shows model calculations where we assume that the dust temperature $T_{\mathrm{d}}$ remains at the mean temperature $\bar{T}$ of the deterministic model (panel 1), while the gas temperature fluctuates stochastically, as in the previous models.

The effects are significant. We note that the assumed rms temperature fluctation $\sigma$ was varied only between $1 \mathrm{~K}$ and $20 \mathrm{~K}$. This calculation represents the upper limit of the possible influence of temperature fluctuations on dust formation; this is because the assumed thermal decoupling between the gas and dust maximises the temperature difference between the gas and dust, having a tremendous effect on the dust growth rates (see e.g. Patzer et al. 1998).

The assumption that a non-fluctuating dust temperature equals the deterministic mean temperature may correspond to assuming that a dust grain is affected by an undisturbed stellar radiation field. This picture appears plausible for large grains in a thin gas. In terms of our model approach, this implies, that the assumption of $T_{\mathrm{d}}=\bar{T}$ might be justified for the net growth rate. The size of the critical cluster, however, will generally not be 


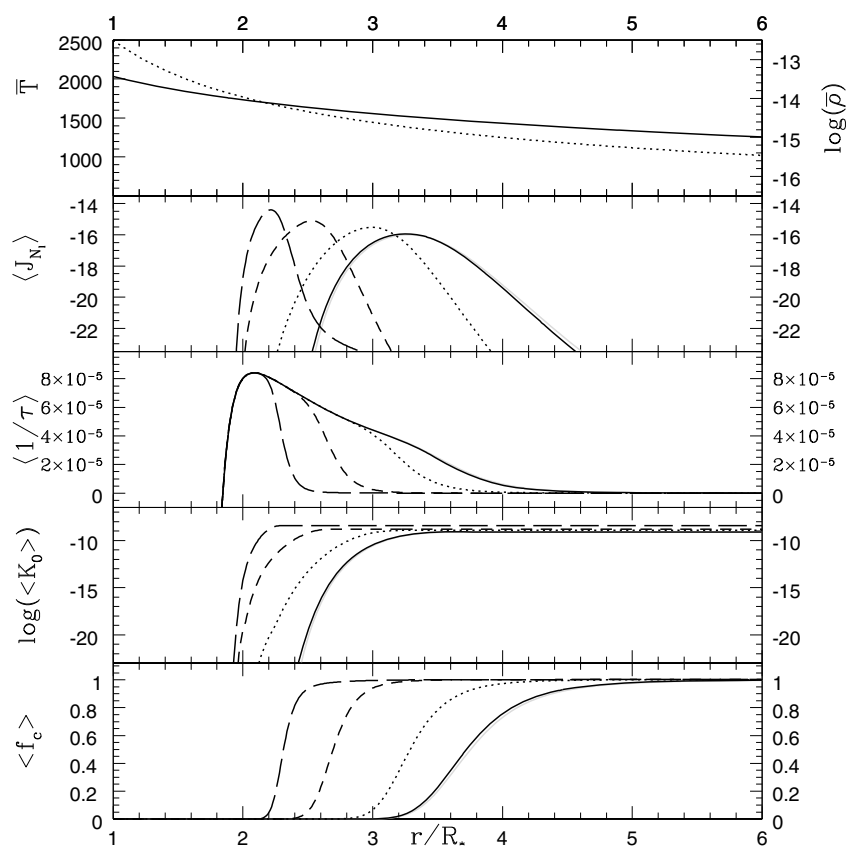

Fig. 5. Parameter study for various mean turbulent temperatures $\sigma, T_{\mathrm{d}}=$ $\bar{T}$. The presented quantities are the same as in Fig. 1. For panels $2-5$ : grey line: deterministic model (close to the full black line), full black line: $\sigma=1 \mathrm{~K}$, dotted: $\sigma=5 \mathrm{~K}$, dashed: $\sigma=10 \mathrm{~K}$, long dashed $\sigma=$ $20 \mathrm{~K} ; \lambda=10^{3} \mathrm{~s}$ for all plots.

large in the regions where remarkable nucleation takes place: we therefore expect this assumption to overestimate the influence of the fluctuation on the nucleation rate.

A realistic calculation of the temperature of the critical cluster would require the solution of the detailed balance between the (frequency-dependent) radiative heating and cooling rates, and the corresponding heating and cooling rates by means of inelastic collisions with the gas.

\section{Dynamical wind models}

To calculate the dynamical wind models, we used the CHILDCode developed by Fleischer et al. (1992). The CHILD-Code is a 1D-hydro code, which solves the coupled equation system of hydrodynamics, thermodynamics, equilibrium chemistry, time-dependent dust nucleation, growth, and evaporation, and grey radiative-transfer with spherical symmetry. Studies using the CHILD-Code were presented by e.g. Fleischer et al. (1992), Winters et al. (1994), Fleischer et al. (1995), Winters et al. (1995), Arndt et al. (1997), Winters et al. (1997), Schirrmacher et al. (2003), and Jeong et al. (2003).

In this work, two model families were calculated. The first model family was calculated using the version of the code described by Winters et al. (1997), whereas for the second model family the version of the code presented by Schirrmacher et al. (2003) was used. The difference between these two approaches relates to the different descriptions of the thermodynamic state and cooling functions. In the version from Winters et al. (1997) (henceforth referred to as model family 1) assumes a monoatomic ideal gas of constant molecular weight and analytical cooling function $\dot{Q}_{\text {rad }} \sim \kappa\left(T_{\text {rad }}^{4}-T_{\text {gas }}^{4}\right)$. In contrast, the second model family (henceforth model family 2) uses tabulated state ${ }^{5}$ and cooling functions $\dot{Q}_{\text {rad }}$, which were calculated

\footnotetext{
5 With state functions, in this work, we refer to the thermal and caloric equations of state.
}

by assuming statistical equilibrium (SE) in the relevant excitational states of the relevant species (for details see Woitke et al. 1996a,b; Schirrmacher et al. 2003).

These two model families were chosen because the results of Sect. 4.4 suggested that differences between the dust and gas temperatures might be of particular importance to assessing the impact of the temperature fluctuations. In the CHILD-Code, the dust temperature is set to be the radiation temperature; the possible differences between gas and dust temperature are therefore sensitive to the cooling behaviour of the gas. Model family 1 was calculated for rapid radiative relaxation towards radiative equilibrium, whereas model family 2 was calculated with a more sophisticated input physics, which allowed for considerable local deviations between gas and radiation temperature.

\subsection{Implementation of the stochastic dust formation into the dynamical wind model}

In the deterministic case, Eqs. (4) are implemented in a straightforward way into the dynamical model calculation for the cases of dust growth and evaporation. The problem of dust destruction, i.e. the shrinking of dust grains by evaporation to sizes below $N_{\ell}$, requires special treatment because the destruction rate is not primarily a function of the state variables of the gas, but depends clearly on the dust distribution function $f(N, t)$ itself. In the CHILD-Code, the dust destruction rate is therefore obtained by means of detailed book-keeping of the dust growth history, as described by Gauger et al. (1990). This procedure cannot be applied to the stochastical treatment presented in Sect. 3, because it is impossible to reconstruct a reasonable grain size distribution function $f(N, t)$ from the conditional probabilities $q_{\rho}$ obtained by the solution of the Fokker-Planck system Eq. (43). This circumstance has to be taken into account when designing an implementation of stochastical dust formation in time-dependent model calculations.

Fortunately, the results presented in Sect. 4 revealed two important features of the implementation of the method into a dynamical framework:

1. the main impact of the temperature fluctuations was seen in the nucleation rate $J$, while the growth and evaporation rate $\tau^{-1}$ remained almost unaffected, - see Fig. 1 and note the logarithmic scale in the plot for $J$ (panel 2), in contrast to the linear scale in the plot for $\tau^{-1}$ (panel 3);

2. on timescales below $10^{4} \mathrm{~s}$, the time evolution of the dust component can be described by the microturbulent limit case. Since the longest time steps occurring in typical model calculations with the CHILD-Code are of the order of several $10^{3} \mathrm{~s}$, a microturbulent description, which is independent of the correlation time $\lambda$ of the fluctuation, is admissible.

Guided by these results, we chose the following approach in implementing the effects of temperature fluctuations in timedependent model calculations. As suggested by the first of the above results, we neglect the influence on the growth and evaporation rate; in addition, we substitute the deterministic nucleation rate by a tabulated stochastic nucleation rate $J_{\text {stoch }}$, obtained by solving the Fokker-Planck system Eq. (43) in a static environment for given gas temperature $T_{\text {gas }}$, density $\rho$, carbon abundance $\epsilon_{\mathrm{C}}$, and rms-temperature deviation $\sigma$ which is the only remaining parameter describing the fluctuation. Figure 7 shows the resulting microturbulent nucleation rates for two different values of $\sigma$ and the deterministic reference (left plot). It can clearly be seen that the dependence on the density is weak, which is a consequence of the normalization of $J$ to the density of hydrogen 
Table 1. Range and spacing of the 3D-tables containing the microturbulent nucleation rates $J_{\text {micro }}$.

\begin{tabular}{ccccc}
\hline \hline & Lower edge & Upper edge & $N$ & Type of spacing \\
\hline$T_{\text {gas }}$ & $700 \mathrm{~K}$ & $2000 \mathrm{~K}$ & 100 & linear \\
$\rho$ & $10^{-18} \mathrm{~g} / \mathrm{cm}^{-3}$ & $10^{-8} \mathrm{~g} / \mathrm{cm}^{-3}$ & 20 & logarithmic \\
$\epsilon_{\mathrm{C}}$ & 0 & $5.3 \times 10^{-4}$ & 50 & linear \\
\hline
\end{tabular}

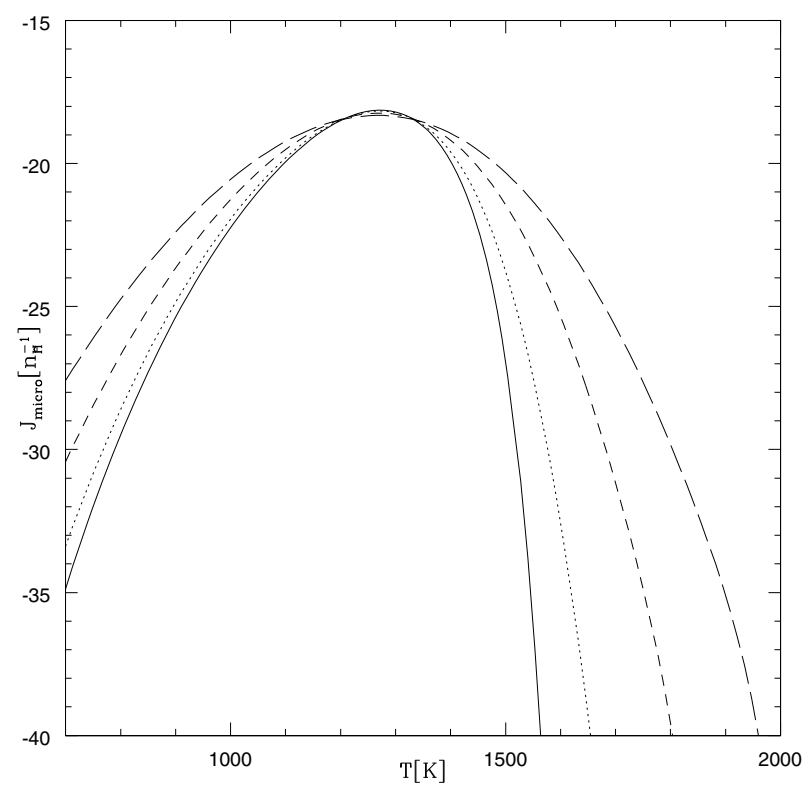

Fig. 6. $\sigma$-study for $J_{\text {micro }}$ for $\rho=10^{-14} \mathrm{~g} / \mathrm{cm}^{-3}$ and $\epsilon_{\mathrm{C}}=5.3 \times 10^{-4}$. $\sigma=1 \mathrm{~K}$ (solid line), $20 \mathrm{~K}$ (dotted), $40 \mathrm{~K}$ (dashed) and $60 \mathrm{~K}$ (long dashed).

cores $n_{\hat{\mathrm{H}}}$. Consequently, the tables were calculated with fewer grid points in the $\rho$-direction (see Table 1). Figure 6 shows slices of the tabulated $J_{\text {micro }}$ for various values of $\sigma$ at typical values for $\rho$ and $\epsilon_{\mathrm{C}}$ during the onset of dust formation in an AGB wind. The peak value of the nucleation rate decreases slightly with increasing fluctuation strength, whereas the onset of nucleation is shifted towards considerably higher temperatures.

There are two main advantages of this approach in implementing the effects of temperature fluctuations in dynamical model calculations:

1. by describing the stochastic dust formation in the microturbulent limit case, the influence of the correlation time $\lambda$ vanishes. This has the advantages that, i) the dimension of the tables for $J_{\text {micro }}$ is reduced by one, which allows superior numerical resolution in the remaining dimensions; and ii) there is no need for a self-consistent description of the correlation times of the fluctuations;

2. by implementing the stochastic nucleation rate, while leaving the growth and evaporation rate, and the equation system Eq. (4) unchanged, the afore mentioned problems of determining the dust destruction rate are evaded, because the average dust destruction is described properly by the deterministic frame of the CHILD-Code.

With respect to dust nucleation and grain destruction, the asymmetry in this approach is justified, because it is intrinsic to the thermodynamics of the dust physics. In an assumed mean thermodynamic situation, if grains of the size of the critical cluster are stable, they might evaporate during a fluctuation, but will reform when the environment relaxes back to its undisturbed
Table 2. Overview over the model parameters of the dynamical wind calculations. $\eta_{\text {opt }}$ is a parameter for the temperature dependence of the dust opacity (see Gail \& Sedlmayr 1987).

\begin{tabular}{rcl}
\hline \hline$M_{\star}\left[M_{\odot}\right]$ & $:$ & $0.8,1.0$ \\
$L_{\star}\left[L_{\odot}\right]$ & $:$ & $6000,8000,10000$ \\
$T_{\star}[\mathrm{K}]$ & $:$ & $2400,2600,2800,3000$ \\
$\eta_{\mathrm{opt}}\left[\mathrm{K}^{-1}\right]$ & $:$ & $4.4,5.9$ \\
$\sigma[\mathrm{K}]$ & $:$ & $0,20,40,60$ \\
\hline$[\mathrm{C} / \mathrm{O}]$ & $:$ & 1.8 \\
$P[$ days $]$ & $:$ & 650 \\
\hline
\end{tabular}

Table 3. Relative changes $\chi_{\sigma}\left(=\dot{M}_{\sigma} / \dot{M}_{\text {det }}\right)$ of the mass-loss rates under the influence of the temperature fluctuations.

\begin{tabular}{lccc}
\hline \hline \multicolumn{4}{c}{$T^{4}$-cooling, ideal gas, $\eta_{\text {opt }}=4.4 \mathrm{~K}^{-1}$} \\
$\sigma$ & $\overline{\chi_{\sigma}}$ & $N_{\text {sample }}$ & $\sigma_{n-1}\left(\chi_{\sigma}\right)$ \\
\hline 20 & 1.07 & 12 & 0.18 \\
40 & 1.11 & 12 & 0.21 \\
60 & 1.18 & 5 & 0.10 \\
\hline \multicolumn{5}{c}{$T^{4}$-cooling, ideal gas, $\eta_{\text {opt }}=5.9 \mathrm{~K}^{-1}$} \\
$\sigma$ & $\overline{\chi_{\sigma}}$ & $N_{\text {sample }}$ & $\sigma_{n-1}\left(\chi_{\sigma}\right)$ \\
\hline 20 & 1.08 & 13 & 0.04 \\
40 & 1.16 & 12 & 0.19 \\
60 & 1.30 & 3 & 0.11 \\
\hline NLTE-cooling and state functions, $\eta_{\text {opt }}=4.4 \mathrm{~K}^{-1}$ \\
$\sigma$ & $\overline{\chi_{\sigma}}$ & $N_{\text {sample }}$ & $\sigma_{n-1}\left(\chi_{\sigma}\right)$ \\
\hline 20 & 0.97 & 19 & 0.11 \\
40 & 1.09 & 19 & 0.26 \\
60 & 1.05 & 12 & 0.31 \\
\hline NLTE-cooling and state functions, $\eta_{\text {opt }}=5.9 \mathrm{~K}^{-1}$ \\
$\sigma$ & $\overline{\chi_{\sigma}}$ & $N_{\text {sample }}$ & $\sigma_{n-1}\left(\chi_{\sigma}\right)$ \\
\hline 20 & 0.92 & 19 & 0.17 \\
40 & 0.97 & 18 & 0.35 \\
60 & 1.03 & 12 & 0.48 \\
\hline \multicolumn{5}{c}{}
\end{tabular}

situation. In a dust-free mean situation, in which nucleation is not allowed, i.e. $1<S<S_{\text {crit }}$, if grains are formed during a temporary temperature deviation, they will not be destroyed, when the undisturbed situation is restored. This situation can occur in the cooling flow of an AGB wind just before deterministic nucleation begins. This earlier (i.e. at higher mean temperatures) onset of nucleation in the presence of temperature fluctuation is described properly by our microturbulent nucleation rate. An overestimation of this effect is avoided, since in the case that the mean thermodynamic conditions leads to an evaporation of macroscopic grains $(S<1)$, the deterministic net growth rate $\tau^{-1}$ will prevent the formation of dust grains.

\subsection{Results of the dynamical model calculations}

A set of over 200 dynamical wind models was calculated. The model parameters are summarized in Table 2. The results of all model calculations are listed in Tables 4 and 5 .

For model family 1 , the models with $T_{\star}=2400 \mathrm{~K}$ (models 6 , 13 , and 16), as well as particularly compact stars $\left(T_{\star}>3000 \mathrm{~K}\right.$, and $L_{\star}<10^{4} M_{\odot}$ ), had numerical problems at the beginning when the first shock front steepened in the hydrostatic start model. The corresponding models are omitted in Table 4.

Model family 2 is calculated using a more sophisticated gas model (see Schirrmacher et al. 2003) based on a statistical equilibrium (SE) calculation of all relevant particle densities and excitation levels of atoms, electrons, molecules, and ions in a 

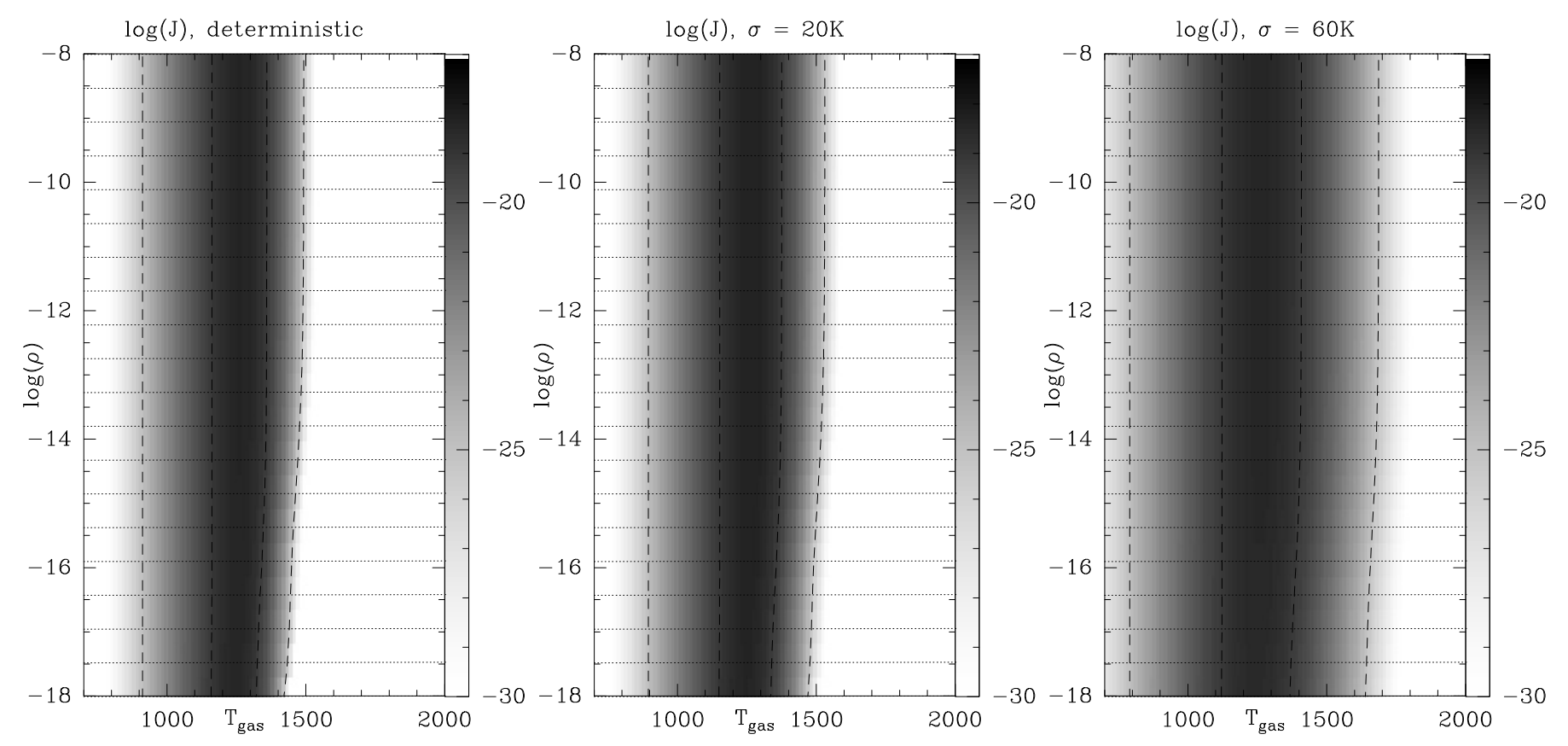

Fig. 7. The microturbulent nucleation rate $\log (J)$ in a $T_{\text {gas }} / \log (\rho)$ plane for $\epsilon_{\mathrm{C}} \approx 5.3 \times 10^{-4}$, i.e. $\mathrm{C} / \mathrm{O} \approx 1.8$. The temperature is given in Kelvin and the density in $\mathrm{g} \mathrm{cm}^{-3}$. The dashed lines correspond to nucleation rates of $\log (J)=-19$ and -25 . The dots correspond to the grid points in the calculation. Left: deterministic rate, middle: $\sigma=20 \mathrm{~K}$, right: $\sigma=60 \mathrm{~K}$.

radiation field parametrised by the radiation temperature $T_{\mathrm{rad}}$, and a local hydrodynamic environment parametrised by an average velocity gradient $\left\langle\frac{\mathrm{d} v}{\mathrm{~d} l}\right\rangle$. These models can show remarkable differences between $T_{\text {gas }}$ and $T_{\text {dust }}$, due to the slower cooling behaviour and inclusion of the dissociation energy of $\mathrm{H}_{2}$ into the caloric equation of state (for details see Schirrmacher et al. 2003).

For models with strong fluctuations $(\sigma=60 \mathrm{~K})$, the calculation was often aborted during the first shock increase in the hydrostatic start model, for both model families. This can usually be avoided by some fine-tuning of the numerical parameters that control the time steps. However, to ensure that we had a coherent sample for the quantitative evaluation, no individual fine-tuning was completed and the corresponding models are omitted or marked with (-) in Tables 4 and 5.

\subsection{Discussion of the dynamical models}

In Fig. 8, a comparison of a typical snapshot of the resulting wind structures for the deterministic and stochastic model is presented. The earlier onset of nucleation in the case including fluctuations (right figure) can be seen in panel 4. However, in these two - as in most other models - the impact of the temperature fluctuations is of a quantitative, rather than qualitative nature. Therefore, a statistical evaluation of the resulting mass loss rates was performed.

The mass loss rate was chosen for the quantitative evaluation, because i) it is the most important wind quantity in the evolution of the star, and the material injection into the surrounding ISM; and ii) it is obtained by averaging the wind structure over a comparably long period of time, so that the impact of numerical noise can be averaged.

The mass loss rates in Tables 4 and 5 were obtained by averaging the mass loss rate at $R=20 R_{\star}$ from periods 90-120. The averaging process was started at $P=90$ to ensure that all disturbances from the starting phase, where the forced pistonoscillation of the velocity at the inner boundary was increased to its final amplitude, and the hydrostatic starting model had to evolve to the quasi-stationary ${ }^{6}$ situation, have had sufficient time to travel to the outer boundary of the model.

In the quantitative evaluation, the ratios between the stochastic and deterministic mass loss rates were calculated according to

$\chi_{20}=\frac{\dot{M}_{\sigma=20 \mathrm{~K}}}{\dot{M}_{\mathrm{det}}}$,

and for $\sigma=40,60 \mathrm{~K}$. These quantities are presented in the rightmost column of Tables 4 and 5.

The $\chi_{\sigma}$ were averaged separately for the two model families and the two different dust extinction coefficients $\eta_{\text {opt }}$. The results are shown in Table 3.

For model family 1 (ideal gas, analytical $T^{4}$-cooling), a clear trend for the mass-loss rates increasing with the fluctuation strength can be seen. This tendency is valid for both values of the dust extinction, but appears to be stronger for the higher values of $\eta_{\mathrm{opt}}$, which is the expected result. The onset of nucleation at lower temperatures facilitates dust formation, resulting in a higher mass loss. The increase in this effect with a stronger coupling of the dust to the accelerating radiation field also appears reasonable. The statistical basis for the values of $\chi_{60}$ is however poor. However, when studying models 10,11 , or 14 , for which the calculation achieved completion for $\sigma=60 \mathrm{~K}$, and $\chi_{60}$ could not be calculated because the deterministic model failed, the trend for mass loss rates increasing with $\sigma$ holds when comparing the corresponding models with $\sigma=20$, or $40 \mathrm{~K}$.

For model family 2 (tabulated SE-state and cooling functions), the results are not as clear. The scattering in the mass-loss rates of the particular models was significantly higher, whereas the differences in the average values of $\chi_{\sigma}$ were smaller. In many cases, the inclusion of the fluctuations even resulted in a decrease in the mass-loss rate, as for 3 of the 6 values of $\chi_{\sigma}$ in Table 3 . We assume that the reason for the far less regular behaviour of

\footnotetext{
6 "Quasi-stationary" refers to the fact that a pulsating star will never have a strictly stationary wind.
} 
Table 4. Mass-loss rates and final velocities for models with $T^{4}$-cooling, ideal gas, (model family 1).

\begin{tabular}{|c|c|c|c|c|c|c|c|c|}
\hline Model & $T_{\star}[\mathrm{K}]$ & $M_{*} / M_{\odot}$ & $L_{*} / L_{\odot}$ & $\eta_{\mathrm{opt}}$ & $\sigma[\mathrm{K}]$ & $v_{\infty}\left[\mathrm{km} \mathrm{s}^{-1}\right]$ & $\dot{M}\left[M_{\odot} / \mathrm{yr}\right]$ & $\chi_{\sigma}=\dot{M}_{\sigma} / \dot{M}_{\mathrm{det}}$ \\
\hline $0 / \mathrm{E}$ & 2600 & 1.0 & $10^{4}$ & $\begin{array}{l}4.4 \\
4.4 \\
4.4 \\
5.9 \\
5.9 \\
5.9\end{array}$ & $\begin{array}{c}- \\
20 \\
40 \\
- \\
20 \\
40\end{array}$ & $\begin{array}{l}26.52 \\
26.28 \\
26.08 \\
29.94 \\
28.87 \\
29.71\end{array}$ & $\begin{array}{l}1.08 \times 10^{-5} \\
1.14 \times 10^{-5} \\
1.21 \times 10^{-5} \\
9.37 \times 10^{-6} \\
9.84 \times 10^{-6} \\
1.08 \times 10^{-5}\end{array}$ & $\begin{array}{l}1.05 \\
1.15\end{array}$ \\
\hline 1 & 2800 & 1.0 & $10^{4}$ & $\begin{array}{l}4.4 \\
4.4 \\
4.4 \\
5.9 \\
5.9 \\
5.9\end{array}$ & $\begin{array}{c}- \\
20 \\
40 \\
- \\
20 \\
40\end{array}$ & $\begin{array}{l}24.45 \\
24.66 \\
25.02 \\
29.50 \\
28.50 \\
28.44\end{array}$ & $\begin{array}{l}8.83 \times 10^{-6} \\
7.80 \times 10^{-6} \\
8.68 \times 10^{-6} \\
7.40 \times 10^{-6} \\
8.11 \times 10^{-6} \\
8.34 \times 10^{-6}\end{array}$ & $\begin{array}{l}0.88 \\
0.98 \\
\\
1.10 \\
1.13\end{array}$ \\
\hline 2 & 3000 & 1.0 & $10^{4}$ & $\begin{array}{l}4.4 \\
4.4 \\
4.4 \\
5.9 \\
5.9 \\
5.9 \\
\end{array}$ & $\begin{array}{c}- \\
20 \\
40 \\
- \\
20 \\
40 \\
\end{array}$ & $\begin{array}{l}21.31 \\
22.57 \\
25.43 \\
26.26 \\
25.30 \\
28.11 \\
\end{array}$ & $\begin{array}{l}4.74 \times 10^{-6} \\
4.94 \times 10^{-6} \\
5.29 \times 10^{-6} \\
4.75 \times 10^{-6} \\
5.20 \times 10^{-6} \\
5.56 \times 10^{-6} \\
\end{array}$ & $\begin{array}{l}1.04 \\
1.12 \\
\\
1.09 \\
1.17 \\
\end{array}$ \\
\hline 3 & 2400 & 1.0 & $10^{4}$ & $\begin{array}{l}4.4 \\
4.4 \\
4.4 \\
5.9 \\
5.9 \\
5.9 \\
\end{array}$ & $\begin{array}{c}- \\
20 \\
40 \\
- \\
20 \\
40\end{array}$ & $\begin{array}{c}23.52 \\
(-) \\
21.25 \\
24.89 \\
24.19 \\
24.32 \\
\end{array}$ & $\begin{array}{c}3.01 \times 10^{-5} \\
(-) \\
3.64 \times 10^{-5} \\
2.91 \times 10^{-5} \\
3.39 \times 10^{-5} \\
3.17 \times 10^{-5}\end{array}$ & $\begin{array}{l}(-) \\
1.21 \\
\\
1.16 \\
1.09 \\
\end{array}$ \\
\hline 4 & 2600 & 1.0 & $8 \times 10^{3}$ & $\begin{array}{l}4.4 \\
4.4 \\
4.4 \\
5.9 \\
5.9 \\
5.9 \\
\end{array}$ & $\begin{array}{c}- \\
20 \\
40 \\
- \\
20 \\
40 \\
\end{array}$ & $\begin{array}{l}23.05 \\
22.66 \\
22.69 \\
26.35 \\
25.88 \\
25.83 \\
\end{array}$ & $\begin{array}{l}9.52 \times 10^{-6} \\
1.06 \times 10^{-5} \\
1.08 \times 10^{-5} \\
9.29 \times 10^{-6} \\
9.80 \times 10^{-6} \\
1.06 \times 10^{-5}\end{array}$ & $\begin{array}{l}1.11 \\
1.13 \\
\\
\\
1.05 \\
1.14 \\
\end{array}$ \\
\hline 5 & 2600 & 1.0 & $6 \times 10^{3}$ & $\begin{array}{l}4.4 \\
4.4 \\
4.4 \\
5.9 \\
5.9 \\
5.9\end{array}$ & $\begin{array}{c}- \\
20 \\
40 \\
- \\
20 \\
40\end{array}$ & $\begin{array}{c}19.39 \\
19.75 \\
(-) \\
23.58 \\
22.97 \\
23.23\end{array}$ & $\begin{array}{c}1.04 \times 10^{-5} \\
7.87 \times 10^{-6} \\
(-) \\
8.00 \times 10^{-6} \\
8.58 \times 10^{-6} \\
7.70 \times 10^{-6}\end{array}$ & $\begin{array}{l}0.76 \\
(-) \\
\\
1.07 \\
0.96\end{array}$ \\
\hline 7 & 2800 & 1.0 & $8 \times 10^{3}$ & $\begin{array}{l}4.4 \\
4.4 \\
4.4 \\
5.9 \\
5.9 \\
5.9 \\
\end{array}$ & $\begin{array}{c}- \\
20 \\
40 \\
- \\
20 \\
40 \\
\end{array}$ & $\begin{array}{l}22.53 \\
21.58 \\
22.84 \\
26.86 \\
25.02 \\
25.61 \\
\end{array}$ & $\begin{array}{l}7.88 \times 10^{-6} \\
8.74 \times 10^{-6} \\
6.62 \times 10^{-6} \\
6.24 \times 10^{-6} \\
6.37 \times 10^{-6} \\
7.42 \times 10^{-6}\end{array}$ & $\begin{array}{l}1.11 \\
0.84 \\
\\
1.02 \\
1.19 \\
\end{array}$ \\
\hline 10 & 2600 & 0.8 & $10^{4}$ & $\begin{array}{l}4.4 \\
4.4 \\
4.4 \\
4.4 \\
5.9 \\
5.9 \\
5.9 \\
5.9\end{array}$ & $\begin{array}{c}- \\
20 \\
40 \\
60 \\
- \\
20 \\
40 \\
60\end{array}$ & $\begin{array}{c}(-) \\
(-) \\
23.96 \\
24.00 \\
(-) \\
26.93 \\
26.63 \\
26.64\end{array}$ & $\begin{array}{c}(-) \\
(-) \\
2.79 \times 10^{-5} \\
2.82 \times 10^{-5} \\
(-) \\
2.43 \times 10^{-5} \\
2.56 \times 10^{-5} \\
2.72 \times 10^{-5}\end{array}$ & $\begin{array}{l}(-) \\
(-) \\
(-) \\
(-) \\
(-)\end{array}$ \\
\hline 11 & 2800 & 0.8 & $10^{4}$ & $\begin{array}{l}4.4 \\
4.4 \\
4.4 \\
4.4 \\
5.9 \\
5.9 \\
5.9 \\
5.9\end{array}$ & $\begin{array}{c}- \\
20 \\
40 \\
60 \\
- \\
20 \\
40 \\
60\end{array}$ & $\begin{array}{c}27.41 \\
26.62 \\
26.40 \\
26.19 \\
(-) \\
30.08 \\
29.81 \\
29.27\end{array}$ & $\begin{array}{c}9.81 \times 10^{-6} \\
1.07 \times 10^{-5} \\
1.11 \times 10^{-5} \\
1.22 \times 10^{-5} \\
(-) \\
9.41 \times 10^{-6} \\
1.04 \times 10^{-5} \\
1.21 \times 10^{-5}\end{array}$ & $\begin{array}{l}1.09 \\
1.13 \\
1.24 \\
\\
(-) \\
(-) \\
(-)\end{array}$ \\
\hline
\end{tabular}


Table 4. continued.

\begin{tabular}{|c|c|c|c|c|c|c|c|c|}
\hline Model & $T_{\star}[\mathrm{K}]$ & $M_{*} / M_{\odot}$ & $L_{*} / L_{\odot}$ & $\eta_{\mathrm{opt}}$ & $\sigma[\mathrm{K}]$ & $v_{\infty}\left[\mathrm{km} \mathrm{s}^{-1}\right]$ & $\dot{M}\left[M_{\odot} / \mathrm{yr}\right]$ & $\chi_{\sigma}=\dot{M}_{\sigma} / \dot{M}_{\mathrm{det}}$ \\
\hline 12 & 3000 & 0.8 & $10^{4}$ & $\begin{array}{l}4.4 \\
4.4 \\
4.4 \\
4.4 \\
5.9 \\
5.9 \\
5.9 \\
5.9 \\
\end{array}$ & $\begin{array}{c}- \\
20 \\
40 \\
60 \\
- \\
20 \\
40 \\
60 \\
\end{array}$ & $\begin{array}{l}24.61 \\
23.92 \\
24.86 \\
24.97 \\
28.28 \\
28.11 \\
27.46 \\
27.40 \\
\end{array}$ & $\begin{array}{l}8.27 \times 10^{-6} \\
8.68 \times 10^{-6} \\
8.88 \times 10^{-6} \\
1.01 \times 10^{-5} \\
7.35 \times 10^{-6} \\
8.40 \times 10^{-6} \\
8.72 \times 10^{-6} \\
9.91 \times 10^{-6}\end{array}$ & $\begin{array}{l}1.05 \\
1.07 \\
1.22 \\
\\
1.14 \\
1.18 \\
1.35 \\
\end{array}$ \\
\hline 14 & 2600 & 0.8 & $8 \times 10^{3}$ & $\begin{array}{l}4.4 \\
4.4 \\
4.4 \\
4.4 \\
5.9 \\
5.9 \\
5.9 \\
5.9\end{array}$ & $\begin{array}{c}- \\
20 \\
40 \\
60 \\
- \\
20 \\
40 \\
60\end{array}$ & $\begin{array}{c}23.92 \\
23.63 \\
24.48 \\
24.41 \\
(-) \\
27.11 \\
26.89 \\
26.80 \\
\end{array}$ & $\begin{array}{c}1.52 \times 10^{-5} \\
1.64 \times 10^{-5} \\
1.39 \times 10^{-5} \\
1.52 \times 10^{-5} \\
(-) \\
1.23 \times 10^{-5} \\
1.29 \times 10^{-5} \\
1.47 \times 10^{-5}\end{array}$ & $\begin{array}{l}1.08 \\
0.91 \\
1.00 \\
\\
(-) \\
(-) \\
(-)\end{array}$ \\
\hline 15 & 2600 & 0.8 & $6 \times 10^{3}$ & $\begin{array}{l}4.4 \\
4.4 \\
4.4 \\
5.9 \\
5.9 \\
5.9 \\
\end{array}$ & $\begin{array}{c}- \\
20 \\
40 \\
- \\
20 \\
40 \\
\end{array}$ & $\begin{array}{c}(-) \\
20.76 \\
20.74 \\
24.12 \\
23.84 \\
23.68 \\
\end{array}$ & $\begin{array}{c}(-) \\
1.21 \times 10^{-5} \\
1.23 \times 10^{-5} \\
9.76 \times 10^{-6} \\
1.05 \times 10^{-5} \\
1.09 \times 10^{-5} \\
\end{array}$ & $\begin{array}{l}(-) \\
(-) \\
\\
1.08 \\
1.12 \\
\end{array}$ \\
\hline 17 & 2800 & 0.8 & $8 \times 10^{3}$ & $\begin{array}{l}4.4 \\
4.4 \\
4.4 \\
4.4 \\
5.9 \\
5.9 \\
5.9 \\
5.9\end{array}$ & $\begin{array}{c}- \\
20 \\
40 \\
60 \\
- \\
20 \\
40 \\
60\end{array}$ & $\begin{array}{l}23.16 \\
22.87 \\
22.79 \\
22.68 \\
27.26 \\
26.74 \\
26.88 \\
26.70 \\
\end{array}$ & $\begin{array}{l}9.22 \times 10^{-6} \\
9.86 \times 10^{-6} \\
1.10 \times 10^{-5} \\
1.14 \times 10^{-5} \\
8.49 \times 10^{-6} \\
9.59 \times 10^{-6} \\
1.02 \times 10^{-5} \\
1.00 \times 10^{-5}\end{array}$ & $\begin{array}{l}1.07 \\
1.19 \\
1.24 \\
\\
1.13 \\
1.20 \\
1.18\end{array}$ \\
\hline 18 & 3000 & 0.8 & $8 \times 10^{3}$ & $\begin{array}{l}4.4 \\
4.4 \\
4.4 \\
4.4 \\
5.9 \\
5.9 \\
5.9 \\
5.9 \\
\end{array}$ & $\begin{array}{c}- \\
20 \\
40 \\
60 \\
- \\
20 \\
40 \\
60 \\
\end{array}$ & $\begin{array}{l}24.38 \\
23.27 \\
23.21 \\
23.93 \\
26.69 \\
25.98 \\
26.08 \\
26.65 \\
\end{array}$ & $\begin{array}{l}4.87 \times 10^{-6} \\
7.57 \times 10^{-6} \\
8.15 \times 10^{-6} \\
5.88 \times 10^{-6} \\
4.26 \times 10^{-6} \\
4.95 \times 10^{-6} \\
7.26 \times 10^{-6} \\
5.87 \times 10^{-6} \\
\end{array}$ & $\begin{array}{l}1.55 \\
1.67 \\
1.21 \\
\\
1.16 \\
1.70 \\
1.38 \\
\end{array}$ \\
\hline 19 & 3000 & 0.8 & $6 \times 10^{3}$ & $\begin{array}{l}4.4 \\
4.4 \\
4.4 \\
5.9 \\
5.9 \\
5.9\end{array}$ & $\begin{array}{c}- \\
20 \\
40 \\
- \\
20 \\
40\end{array}$ & $\begin{array}{l}19.63 \\
19.36 \\
20.70 \\
23.65 \\
22.79 \\
23.20\end{array}$ & $\begin{array}{l}8.37 \times 10^{-7} \\
8.67 \times 10^{-7} \\
7.61 \times 10^{-7} \\
7.47 \times 10^{-7} \\
8.33 \times 10^{-7} \\
7.27 \times 10^{-7}\end{array}$ & $\begin{array}{l}1.04 \\
0.91\end{array}$ \\
\hline
\end{tabular}

All models in this table were calculated for a C/O-ratio of 1.8, a pulsation period of 650 days, and a piston velocity of $2 \mathrm{~km} \mathrm{~s}^{-1}$.

this model family is that the coupling in the equation system of the dynamical formulation has a stronger non-linear character than for model family 1 . Effects such as the dissociation of hydrogen, and the dependence of the SE-radiative cooling rates on the chemical state of the gas are included in model family 2 (see Schirrmacher et al. 2003), and induce a strong non-linear behaviour, so that a clear trend in the influence of the temperature fluctuations on these models cannot be extracted from our calculations.

\section{Conclusions}

We have presented a time-dependent stochastic formulation of astrophysical dust formation under the influence of temperature fluctuations and a method to apply this to dynamical model calculations of dust-driven winds of AGB stars.

The formulation of the dust formation process by means of the theory of stochastic processes has first produced a set of coupled Fokker-Plack equations replacing the deterministic dust moment equations. Since this formulation was technically not yet suited to the direct implementation in a dynamical wind calculation, we have investigated a set of gas-box models to determine i) the maximum timescale of correlation time $\lambda$ that would prohibit a microturbulent formulation of the stochastic dust formation; and ii) the order of the rms-temperature deviation $\sigma$, for which a remarkable influence of the fluctuations on the dust formation can be expected. For values of $\lambda<10^{4} \mathrm{~s}$, the microturbulent approximation was found to be valid, and for values 
Table 5. Mass-loss rates and final velocities, models with tabulated NLTE-cooling and state functions, (model family 2).

\begin{tabular}{|c|c|c|c|c|c|c|c|c|}
\hline Model & $T_{\star}[\mathrm{K}]$ & $M_{*} / M_{\odot}$ & $L_{*} / L_{\odot}$ & $\eta_{\mathrm{opt}}$ & $\sigma[\mathrm{K}]$ & $v_{\infty}\left[\mathrm{km} \mathrm{s}^{-1}\right]$ & $\dot{M}\left[M_{\odot} / \mathrm{yr}\right]$ & $\chi_{\sigma}=\dot{M}_{\sigma} / \dot{M}_{\mathrm{det}}$ \\
\hline $0 / \mathrm{E}$ & 2600 & 1.0 & $10^{4}$ & $\begin{array}{l}4.4 \\
4.4 \\
4.4 \\
4.4 \\
5.9 \\
5.9 \\
5.9 \\
5.9\end{array}$ & $\begin{array}{c}- \\
20 \\
40 \\
60 \\
- \\
20 \\
40 \\
60\end{array}$ & $\begin{array}{l}24.35 \\
25.04 \\
24.55 \\
24.52 \\
27.50 \\
27.35 \\
27.62 \\
27.47\end{array}$ & $\begin{array}{l}1.87 \times 10^{-5} \\
1.82 \times 10^{-5} \\
1.69 \times 10^{-5} \\
1.63 \times 10^{-5} \\
1.73 \times 10^{-5} \\
1.68 \times 10^{-5} \\
1.78 \times 10^{-5} \\
1.89 \times 10^{-5}\end{array}$ & $\begin{array}{l}0.97 \\
1.03 \\
1.13\end{array}$ \\
\hline 1 & 2800 & 1.0 & $10^{4}$ & $\begin{array}{l}4.4 \\
4.4 \\
4.4 \\
4.4 \\
5.9 \\
5.9 \\
5.9 \\
5.9 \\
\end{array}$ & $\begin{array}{c}- \\
20 \\
40 \\
60 \\
- \\
20 \\
40 \\
60 \\
\end{array}$ & $\begin{array}{l}23.76 \\
24.11 \\
24.34 \\
24.45 \\
26.76 \\
27.06 \\
28.27 \\
29.99 \\
\end{array}$ & $\begin{array}{l}6.86 \times 10^{-6} \\
6.17 \times 10^{-6} \\
8.94 \times 10^{-6} \\
1.01 \times 10^{-5} \\
1.01 \times 10^{-5} \\
7.01 \times 10^{-6} \\
7.48 \times 10^{-6} \\
4.68 \times 10^{-6}\end{array}$ & $\begin{array}{l}0.90 \\
1.30 \\
1.47 \\
\\
0.70 \\
0.74 \\
0.46 \\
\end{array}$ \\
\hline 2 & 3000 & 1.0 & $10^{4}$ & $\begin{array}{l}4.4 \\
4.4 \\
4.4 \\
4.4 \\
5.9 \\
5.9 \\
5.9 \\
5.9 \\
\end{array}$ & $\begin{array}{c}- \\
20 \\
40 \\
60 \\
- \\
20 \\
40 \\
60 \\
\end{array}$ & $\begin{array}{l}21.91 \\
23.97 \\
26.36 \\
26.12 \\
26.01 \\
29.05 \\
28.83 \\
29.09 \\
\end{array}$ & $\begin{array}{l}2.67 \times 10^{-6} \\
2.46 \times 10^{-6} \\
3.83 \times 10^{-6} \\
4.05 \times 10^{-6} \\
2.02 \times 10^{-6} \\
2.78 \times 10^{-6} \\
2.93 \times 10^{-6} \\
4.37 \times 10^{-6} \\
\end{array}$ & $\begin{array}{l}0.92 \\
1.43 \\
1.51 \\
\\
1.38 \\
1.45 \\
2.16 \\
\end{array}$ \\
\hline 3 & 2400 & 1.0 & $10^{4}$ & $\begin{array}{l}4.4 \\
4.4 \\
4.4 \\
5.9 \\
5.9 \\
5.9\end{array}$ & $\begin{array}{c}- \\
20 \\
40 \\
- \\
20 \\
40\end{array}$ & $\begin{array}{l}22.64 \\
22.91 \\
23.31 \\
26.14 \\
27.00 \\
26.92\end{array}$ & $\begin{array}{l}2.89 \times 10^{-5} \\
2.70 \times 10^{-5} \\
2.71 \times 10^{-5} \\
3.29 \times 10^{-5} \\
2.30 \times 10^{-5} \\
2.06 \times 10^{-5}\end{array}$ & $\begin{array}{l}0.93 \\
0.94 \\
\\
0.70 \\
0.63 \\
\end{array}$ \\
\hline 4 & 2600 & 1.0 & $8 \times 10^{3}$ & $\begin{array}{l}4.4 \\
4.4 \\
4.4 \\
5.9 \\
5.9 \\
5.9\end{array}$ & $\begin{array}{c}- \\
20 \\
40 \\
- \\
20 \\
40\end{array}$ & $\begin{array}{l}23.54 \\
23.30 \\
24.90 \\
28.28 \\
28.71 \\
28.67\end{array}$ & $\begin{array}{l}4.86 \times 10^{-6} \\
5.47 \times 10^{-6} \\
3.87 \times 10^{-6} \\
3.95 \times 10^{-6} \\
4.00 \times 10^{-6} \\
3.07 \times 10^{-6}\end{array}$ & $\begin{array}{l}1.13 \\
0.80 \\
\\
\\
1.01 \\
0.78\end{array}$ \\
\hline 5 & 2600 & 1.0 & $6 \times 10^{3}$ & $\begin{array}{l}4.4 \\
4.4 \\
4.4 \\
5.9 \\
5.9 \\
5.9 \\
\end{array}$ & $\begin{array}{c}- \\
20 \\
40 \\
- \\
20 \\
40 \\
\end{array}$ & $\begin{array}{l}20.53 \\
19.86 \\
20.89 \\
23.85 \\
24.22 \\
23.30 \\
\end{array}$ & $\begin{array}{l}3.20 \times 10^{-6} \\
2.76 \times 10^{-6} \\
4.47 \times 10^{-6} \\
3.45 \times 10^{-6} \\
3.45 \times 10^{-6} \\
3.54 \times 10^{-6}\end{array}$ & $\begin{array}{l}0.86 \\
1.40 \\
\\
\\
1.00 \\
1.03\end{array}$ \\
\hline 6 & 2400 & 1.0 & $8 \times 10^{3}$ & $\begin{array}{l}4.4 \\
4.4 \\
4.4 \\
5.9 \\
5.9 \\
5.9 \\
\end{array}$ & $\begin{array}{c}- \\
20 \\
40 \\
- \\
20 \\
40 \\
\end{array}$ & $\begin{array}{l}22.41 \\
22.29 \\
22.15 \\
25.36 \\
25.65 \\
25.18 \\
\end{array}$ & $\begin{array}{l}2.16 \times 10^{-5} \\
2.21 \times 10^{-5} \\
2.32 \times 10^{-5} \\
1.93 \times 10^{-5} \\
1.89 \times 10^{-5} \\
2.22 \times 10^{-5} \\
\end{array}$ & $\begin{array}{l}1.02 \\
1.07 \\
\\
0.98 \\
1.15 \\
\end{array}$ \\
\hline 7 & 2800 & 1.0 & $8 \times 10^{3}$ & $\begin{array}{l}4.4 \\
4.4 \\
4.4 \\
5.9 \\
5.9 \\
5.9 \\
\end{array}$ & $\begin{array}{c}- \\
20 \\
40 \\
- \\
20 \\
40 \\
\end{array}$ & $\begin{array}{l}22.61 \\
23.73 \\
23.51 \\
25.93 \\
26.00 \\
25.30 \\
\end{array}$ & $\begin{array}{l}4.00 \times 10^{-6} \\
3.50 \times 10^{-6} \\
5.30 \times 10^{-6} \\
4.79 \times 10^{-6} \\
4.79 \times 10^{-6} \\
3.67 \times 10^{-6} \\
\end{array}$ & $\begin{array}{l}0.88 \\
1.33 \\
\\
\\
1.00 \\
0.77 \\
\end{array}$ \\
\hline 8 & 3000 & 1.0 & $8 \times 10^{3}$ & $\begin{array}{l}4.4 \\
4.4 \\
4.4 \\
5.9 \\
5.9 \\
5.9\end{array}$ & $\begin{array}{c}- \\
20 \\
40 \\
- \\
20 \\
40\end{array}$ & $\begin{array}{l}19.16 \\
18.32 \\
17.41 \\
21.05 \\
19.16 \\
20.71\end{array}$ & $\begin{array}{l}2,72 \times 10^{-7} \\
2.84 \times 10^{-7} \\
4.34 \times 10^{-7} \\
3.07 \times 10^{-7} \\
3.17 \times 10^{-7} \\
4.99 \times 10^{-7}\end{array}$ & $\begin{array}{l}1.04 \\
1.60\end{array}$ \\
\hline
\end{tabular}


Table 5. continued.

\begin{tabular}{|c|c|c|c|c|c|c|c|c|}
\hline Model & $T_{\star}[\mathrm{K}]$ & $M_{*} / M_{\odot}$ & $L_{*} / L_{\odot}$ & $\eta_{\mathrm{opt}}$ & $\sigma[\mathrm{K}]$ & $v_{\infty}\left[\mathrm{km} \mathrm{s}^{-1}\right]$ & $\dot{M}\left[M_{\odot} / \mathrm{yr}\right]$ & $\chi_{\sigma}=\dot{M}_{\sigma} / \dot{M}_{\mathrm{det}}$ \\
\hline \multirow[t]{6}{*}{9} & 3000 & 1.0 & $6 \times 10^{3}$ & 4.4 & - & 13.00 & $8.24 \times 10^{-8}$ & \\
\hline & & & & 4.4 & 20 & 12.99 & $8.30 \times 10^{-8}$ & 1.00 \\
\hline & & & & 4.4 & 40 & 13.03 & $8.20 \times 10^{-8}$ & 1.00 \\
\hline & & & & 5.9 & - & $(-)$ & $(-)$ & \\
\hline & & & & 5.9 & 20 & 15.04 & $6.78 \times 10^{-8}$ & $(-)$ \\
\hline & & & & 5.9 & 40 & 15.09 & $6.77 \times 10^{-8}$ & $(-)$ \\
\hline \multirow[t]{8}{*}{10} & 2600 & 0.8 & $10^{4}$ & 4.4 & - & 26.32 & $1.19 \times 10^{-5}$ & \\
\hline & & & & 4.4 & 20 & 26.89 & $1.07 \times 10^{-5}$ & 0.90 \\
\hline & & & & 4.4 & 40 & 24.30 & $1.78 \times 10^{-5}$ & 1.50 \\
\hline & & & & 4.4 & 60 & 24.65 & $1.85 \times 10^{-5}$ & 1.55 \\
\hline & & & & 5.9 & - & 28.89 & $1.15 \times 10^{-5}$ & \\
\hline & & & & 5.9 & 20 & 29.54 & $1.04 \times 10^{-5}$ & 0.90 \\
\hline & & & & 5.9 & 40 & 27.10 & $1.77 \times 10^{-5}$ & 1.54 \\
\hline & & & & 5.9 & 60 & 27.73 & $1.76 \times 10^{-5}$ & 1.53 \\
\hline \multirow[t]{8}{*}{11} & 2800 & 0.8 & $10^{4}$ & 4.4 & - & 24.02 & $8.40 \times 10^{-6}$ & \\
\hline & & & & 4.4 & 20 & 25.31 & $1.05 \times 10^{-5}$ & 1.25 \\
\hline & & & & 4.4 & 40 & 25.78 & $7.06 \times 10^{-6}$ & 0.84 \\
\hline & & & & 4.4 & 60 & 26.52 & $7.22 \times 10^{-6}$ & 0.86 \\
\hline & & & & 5.9 & - & 28.51 & $6.99 \times 10^{-6}$ & \\
\hline & & & & 5.9 & 20 & 28.19 & $4.93 \times 10^{-6}$ & 0.71 \\
\hline & & & & 5.9 & 40 & 28.53 & $9.06 \times 10^{-6}$ & 1.30 \\
\hline & & & & 5.9 & 60 & 29.64 & $5.05 \times 10^{-6}$ & 0.72 \\
\hline \multirow[t]{8}{*}{12} & 3000 & 0.8 & $10^{4}$ & 4.4 & - & 25.53 & $9.22 \times 10^{-6}$ & \\
\hline & & & & 4.4 & 20 & 25.41 & $8.90 \times 10^{-6}$ & 0.97 \\
\hline & & & & 4.4 & 40 & 25.73 & $9.88 \times 10^{-6}$ & 1.07 \\
\hline & & & & 4.4 & 60 & 25.90 & $8.62 \times 10^{-6}$ & 0.93 \\
\hline & & & & 5.9 & - & 27.55 & $8.54 \times 10^{-6}$ & \\
\hline & & & & 5.9 & 20 & 27.77 & $8.23 \times 10^{-6}$ & 0.96 \\
\hline & & & & 5.9 & 40 & 29.04 & $7.50 \times 10^{-6}$ & 0.88 \\
\hline & & & & 5.9 & 60 & 28.76 & $6.62 \times 10^{-6}$ & 0.78 \\
\hline \multirow[t]{8}{*}{13} & 2400 & 0.8 & $10^{4}$ & 4.4 & - & 22.48 & $3.98 \times 10^{-5}$ & \\
\hline & & & & 4.4 & 20 & 23.92 & $2.97 \times 10^{-5}$ & 0.75 \\
\hline & & & & 4.4 & 40 & 22.19 & $4.60 \times 10^{-5}$ & 1.16 \\
\hline & & & & 4.4 & 60 & 23.70 & $3.26 \times 10^{-5}$ & 0.82 \\
\hline & & & & 5.9 & - & 24.68 & $5.27 \times 10^{-5}$ & \\
\hline & & & & 5.9 & 20 & 25.79 & $3.41 \times 10^{-5}$ & 0.65 \\
\hline & & & & 5.9 & 40 & $(-)$ & $(-)$ & $(-)$ \\
\hline & & & & 5.9 & 60 & 25.75 & $3.27 \times 10^{-5}$ & 0.62 \\
\hline \multirow[t]{8}{*}{14} & 2600 & 0.8 & $8 \times 10^{3}$ & 4.4 & - & 22.73 & $1.27 \times 10^{-5}$ & \\
\hline & & & & 4.4 & 20 & 22.71 & $1.35 \times 10^{-5}$ & 1.06 \\
\hline & & & & 4.4 & 40 & 23.49 & $9.85 \times 10^{-6}$ & 0.78 \\
\hline & & & & 4.4 & 60 & 23.08 & $1.39 \times 10^{-5}$ & 1.09 \\
\hline & & & & 5.9 & - & 26.70 & $1.12 \times 10^{-5}$ & \\
\hline & & & & 5.9 & 20 & 26.47 & $1.08 \times 10^{-5}$ & 0.96 \\
\hline & & & & 5.9 & 40 & 26.53 & $1.00 \times 10^{-5}$ & 0.89 \\
\hline & & & & 5.9 & 60 & 26.32 & $8.58 \times 10^{-6}$ & 0.77 \\
\hline \multirow[t]{6}{*}{15} & 2600 & 0.8 & $6 \times 10^{3}$ & 4.4 & - & 20.49 & $9.94 \times 10^{-6}$ & \\
\hline & & & & 4.4 & 20 & 20.56 & $9.46 \times 10^{-6}$ & 0.95 \\
\hline & & & & 4.4 & 40 & 20.46 & $8.13 \times 10^{-6}$ & 0.82 \\
\hline & & & & 5.9 & - & 23.61 & $9.35 \times 10^{-6}$ & \\
\hline & & & & 5.9 & 20 & 23.32 & $9.84 \times 10^{-6}$ & 1.05 \\
\hline & & & & 5.9 & 40 & 24.74 & $4.38 \times 10^{-6}$ & 0.47 \\
\hline \multirow[t]{8}{*}{16} & 2400 & 0.8 & $8 \times 10^{3}$ & 4.4 & - & $(-)$ & $(-)$ & \\
\hline & & & & 4.4 & 20 & 22.72 & $2.14 \times 10^{-5}$ & $(-)$ \\
\hline & & & & 4.4 & 40 & 22.27 & $2.25 \times 10^{-5}$ & $(-)$ \\
\hline & & & & 4.4 & 60 & 22.71 & $2.09 \times 10^{-5}$ & $(-)$ \\
\hline & & & & 5.9 & - & 25.58 & $2.15 \times 10^{-5}$ & \\
\hline & & & & 5.9 & 20 & 25.43 & $1.96 \times 10^{-5}$ & 0.91 \\
\hline & & & & 5.9 & 40 & 25.65 & $2.09 \times 10^{-5}$ & 0.97 \\
\hline & & & & 5.9 & 60 & 25.20 & $2.40 \times 10^{-5}$ & 1.12 \\
\hline
\end{tabular}


Table 5. continued.

\begin{tabular}{|c|c|c|c|c|c|c|c|c|}
\hline Model & $T_{\star}[\mathrm{K}]$ & $M_{*} / M_{\odot}$ & $L_{*} / L_{\odot}$ & $\eta_{\mathrm{opt}}$ & $\sigma[\mathrm{K}]$ & $v_{\infty}\left[\mathrm{km} \mathrm{s}^{-1}\right]$ & $\dot{M}\left[M_{\odot} / \mathrm{yr}\right]$ & $\chi_{\sigma}=\dot{M}_{\sigma} / \dot{M}_{\mathrm{de}}$ \\
\hline \multirow[t]{8}{*}{17} & 2800 & 0.8 & $8 \times 10^{3}$ & 4.4 & - & 22.50 & $9.26 \times 10^{-6}$ & \\
\hline & & & & 4.4 & 20 & 22.93 & $8.67 \times 10^{-6}$ & 0.94 \\
\hline & & & & 4.4 & 40 & 22.80 & $8.78 \times 10^{-6}$ & 0.95 \\
\hline & & & & 4.4 & 60 & 23.86 & $7.40 \times 10^{-6}$ & 0.80 \\
\hline & & & & 5.9 & - & 25.90 & $7.49 \times 10^{-6}$ & \\
\hline & & & & 5.9 & 20 & 25.84 & $7.10 \times 10^{-6}$ & 0.95 \\
\hline & & & & 5.9 & 40 & 26.87 & $7.13 \times 10^{-6}$ & 0.96 \\
\hline & & & & 5.9 & 60 & 27.03 & $6.91 \times 10^{-6}$ & 0.92 \\
\hline \multirow[t]{8}{*}{18} & 3000 & 0.8 & $8 \times 10^{3}$ & 4.4 & - & 22.52 & $4.05 \times 10^{-6}$ & \\
\hline & & & & 4.4 & 20 & 24.57 & $3.76 \times 10^{-6}$ & 0.93 \\
\hline & & & & 4.4 & 40 & 24.63 & $3.94 \times 10^{-6}$ & 0.97 \\
\hline & & & & 4.4 & 60 & 23.80 & $3.60 \times 10^{-6}$ & 0.89 \\
\hline & & & & 5.9 & - & 25.52 & $3.80 \times 10^{-6}$ & \\
\hline & & & & 5.9 & 20 & 26.61 & $2.74 \times 10^{-6}$ & 0.72 \\
\hline & & & & 5.9 & 40 & 26.74 & $3.73 \times 10^{-6}$ & 0.98 \\
\hline & & & & 5.9 & 60 & 25.80 & $4.16 \times 10^{-6}$ & 1.09 \\
\hline \multirow[t]{6}{*}{19} & 3000 & 0.8 & $6 \times 10^{3}$ & 4.4 & - & 19.94 & $5.40 \times 10^{-7}$ & \\
\hline & & & & 4.4 & 20 & 20.18 & $5.03 \times 10^{-7}$ & 0.93 \\
\hline & & & & 4.4 & 40 & 22.10 & $4.87 \times 10^{-7}$ & 0.90 \\
\hline & & & & 5.9 & - & 25.69 & $1.72 \times 10^{-6}$ & \\
\hline & & & & 5.9 & 20 & 25.87 & $1.71 \times 10^{-6}$ & 0.99 \\
\hline & & & & 5.9 & 40 & 23.74 & $5.21 \times 10^{-7}$ & 0.30 \\
\hline
\end{tabular}

All models in this table were calculated for a C/O-ratio of 1.8, a pulsation period of 650 days, and a piston velocity of $2 \mathrm{~km} \mathrm{~s}^{-1}$.
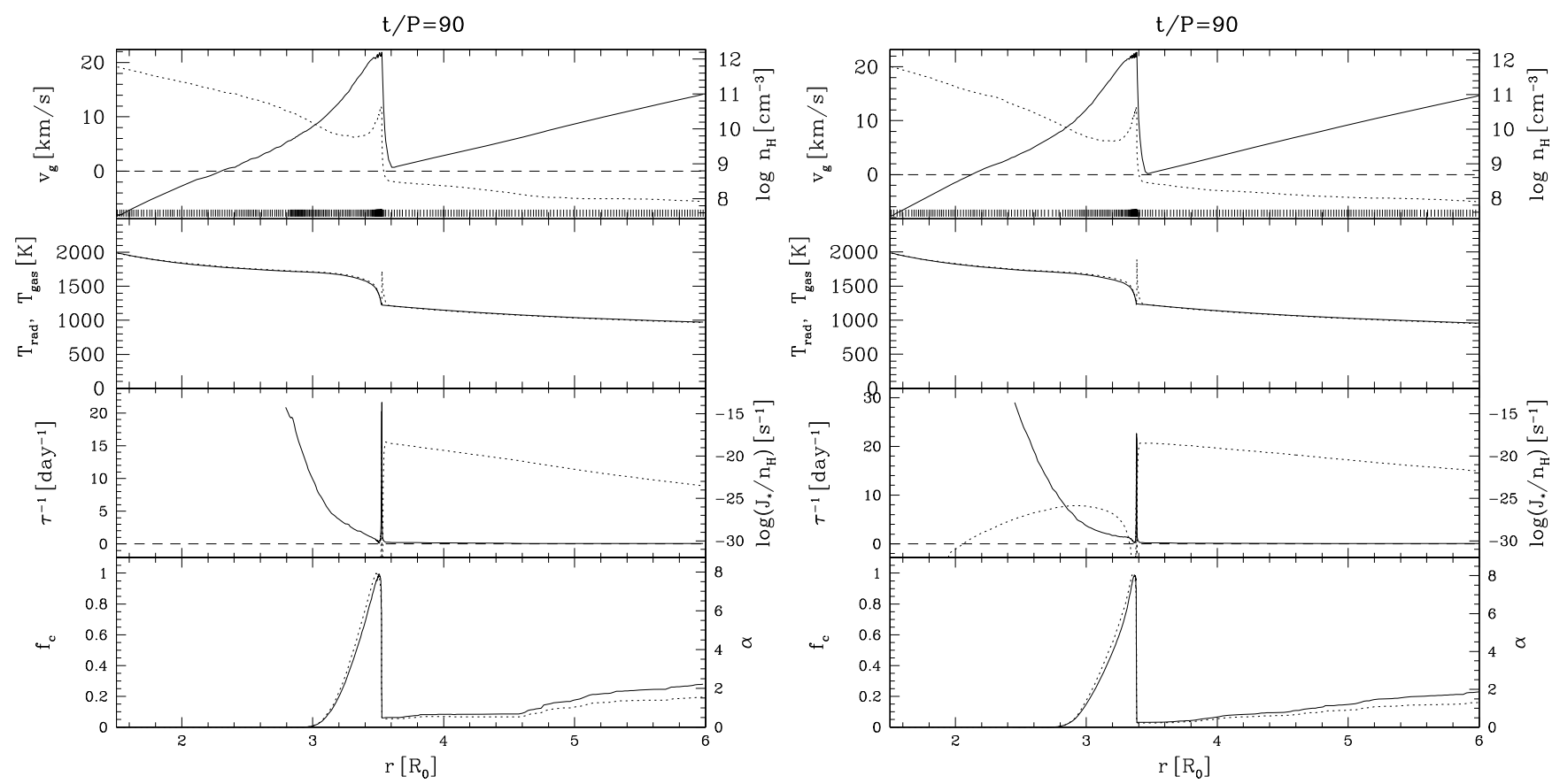

Fig. 8. Snapshots from the dynamical model calculations at $t=90 P$, model 12 , NLTE-cooling, $\eta_{\mathrm{opt}}=4.4 \mathrm{~K}^{-1}$. Left: deterministic, right: $\sigma=60 \mathrm{~K}$. Represented quantities: top panel: velocity $v$ (solid), particle density of hydrogen cores $n_{\hat{\mathrm{H}}}$ (dotted), the small ticks indicate the positions of the grid points. Panel 2: gas temperature $T_{\text {gas }}$ (dotted), radiation temperature $T_{\text {rad }}$ (solid). Panel 3: nucleation rate $J$ (dotted), net growth rate $\tau^{-1}$ (solid). Panel 4: degree of condensation $f_{\mathrm{c}}$ (solid), radiative acceleration $\alpha$ (dotted).

of $\sigma \gtrsim 20 \mathrm{~K}$, the influence of the temperature fluctuations on the dust formation was remarkable. The main influence of the temperature fluctuations on the dust formation was the fluctuation-induced nucleation in a region that remained dustfree in the deterministic case. Effective dust formation therefore occurred for a supersaturation ratio $S \approx 1$, i.e. the critical supersaturation ratio was lowered.
Guided by the results of these gas-box calculations we proposed a microturbulent approach, easily implemented on a subgrid level into dynamical model-calculations. A set of numerical model calculations using this microturbulent sub-grid model for the temperature fluctuations was also presented. The results showed a clear trend of massloss rates increasing with increasing fluctuation strength, close to LTE. Model calculations using 
a more sophisticated gas description with tabulated SE-state and cooling functions were too unreliable to establish such a trend.

The application of the presented microturbulent nucleation rate to other numerical codes for AGB winds is straightforward, as long as the deterministic code uses the moments of the dust distribution function for the description of the time evolution of the dust component.

The presented method for the stochastical description of dust formation under the influence of temperature fluctuations can be applied to other astrophysical situations, if a deterministic timedependent formulation of the dust physics, based on moments of the grain-size distribution function, is available, which can be used for the determination of the transition probabilities and possibly as start and boundary values of the resulting Fokker-Planck system. To apply a microturbulent nucleation rate to other dustforming astrophysical systems, it is necessary to investigate first the importance and timescales of correlation effects. This investigation could be completed using the methods presented in Sect. 4.

For completeness we make two further remarks:

(i) More special physical assumptions about the temperature equalization behaviour after fluctuation displacement could be formulated in the first summand of the stochastic differential equation Eq. (20), which as a result produces other stochastic processes, i.e. provides a description of the fluctuation case.

(ii) The fluctuation-induced increase in the nucleation rate depends critically on possible temperature differences between the critical clusters and the surrounding gas. However, a realistic modelling of the cluster temperature in a dynamical environment is difficult, because a detailed knowledge of the exact nucleation path is required and the cross-sections for inelastic gas-cluster collisions and frequency-dependent cluster opacities are unavailable at present.

Acknowledgements. The members of the Centre of Astronomy and Astrophysics (TU Berlin) are thanked for many fruitful discussions on the subject. In particular Dr. J. M. Winters is thanked for useful advises at the beginning of the project and Dr. B. Patzer, Dr. M. Hegmann, and Prof. Dr. W. Kegel for valuable comments. This work was partly supported by the DFG-SFB 555 .

\section{References}

Arndt, T. U., Fleischer, A. J., \& Sedlmayr, E. 1997, A\&A, 327, 614 Arnold, L. 1974, Stochastic Differential Equations (John Wiley \& Sons)
Bharucha-Reid, A. T. 1960, Elements of the Theory of Markov Processes and Their Applications (Almqvist \& Wiksell International)

Bowen, G. H. 1988, ApJ, 329, 299

Dirks, U. 1993, Master's thesis, Technische Universität Berlin, Germany

Dirks, U. 2000, Ph.D. Thesis, Technische Universität Berlin, Germany,

(http://edocs.tu-berlin.de/diss/2000/dirks_ulrich.htm)

Dirks, U., \& Sedlmayr, E. 1998, in AGB-Stars, ed. T. Le Bertre, A. Lèbre, \& C. Waelkens, IAU Symp. 191, poster P3-03

Dorfi, E. A., \& Höfner, S. 1991, A\&A, 248, 105

Fleischer, A. J., Gauger, A., \& Sedlmayr, E. 1992, A\&A, 266, 321

Fleischer, A. J., Gauger, A., \& Sedlmayr, E. 1995, A\&A, 297, 543

Gail, H.-P., \& Sedlmayr, E. 1974, A\&A, 36, 17

Gail, H.-P., \& Sedlmayr, E. 1987, A\&A, 177, 186

Gail, H.-P., \& Sedlmayr, E. 1988, A\&A, 206, 153

Gail, H.-P., Hundt, E., Kegel, W. H., Schmidt-Burgk, J., \& Traving, G. 1974, A\&A, 32, 65

Gail, H.-P., Sedlmayr, E., \& Traving, G. 1980, JQSRT, 23, 267

Gail, H.-P., Keller, R., \& Sedlmayr, E. 1984, A\&A, 133, 320

Gauger, A., Gail, H.-P., \& Sedlmayr, E. 1990, A\&A, 235, 345

Gautschy-Loidl, R., Höfner, S., Jørgensen, U. G., \& Hron, J. 2004, A\&A, 422, 289

Gel'fand, I. M., \& Vilenkin, N. Y. 1964, Generalized Functions, 4 (Academic Press)

Helling, C., \& Woitke, P. 2006, A\&A, 455, 325

Helling, C., Oevermann, M., Lüttke, M. J. H., Klein, R., \& Sedlmayr, E. 2001, A\&A, 376, 194

Höfner, S., Fleischer, A. J., Gauger, A., et al. 1996, A\&A, 314, 204

Jeong, K., Winters, J., Le Bertre, T., \& Sedlmayr, E. 2003, A\&A, 407, 191

Patzer, A. B. C., Gauger, A., \& Sedlmayr, E. 1998, A\&A, 337

Schirrmacher, V. 2007, Ph.D. Thesis, Technische Universität Berlin, Germany, (http://opus.kobv.de/tuberlin/volltexte/2007/1691/)

Schirrmacher, V., \& Dirks, U. 2003, Astron. Notes, 324, Suppl. Issue 3, 128

Schirrmacher, V., Woitke, P., \& Sedlmayr, E. 2003, A\&A, 404, 267

Schirrmacher, V., Dirks, U., \& Sedlmayr, E. 2004, Astron. Notes, 325, Suppl. Issue 1, 26

Schirrmacher, V., Dirks, U., \& Sedlmayr, E. 2005, in XXV Dynamic Days Europe 2005 (Book of Abstracts), 182

Sedlmayr, E., \& Winters, J. M. 1997, in Stellar Atmospheres: Theory and Observations, ed. J. P. De Greve, R. Blomme, \& H. Hensberge (Springer), Lect. Notes in Phys., 497, 89

Todorovic, P. 1992, An Introduction to Stochastic Processes and Their Applications (Springer-Verlag)

Traving, G. 1975, in Problems in Stellar Atmospheres and Envelopes, ed. B. Baschek, W. Kegel, \& G. Traving, 326

Winters, J. M., Fleischer, A. J., Gauger, A., \& Sedlmayr, E. 1994, A\&A, 290, 623

Winters, J. M., Fleischer, A. J., Gauger, A., \& Sedlmayr, E. 1995, A\&A, 302, 483

Winters, J. M., Fleischer, A. J., Le Bertre, T., \& Sedlmayr, E. 1997, A\&A, 326, 305

Winters, J. M., Le Bertre, T., Jeong, K. S., Helling, C., \& Sedlmayr, E. 2000, A\&A, 361, 641

Woitke, P. 2006, A\&A, 452, 537

Woitke, P., Goeres, A., \& Sedlmayr, E. 1996a, A\&A, 313, 217

Woitke, P., Krüger, D., \& Sedlmayr, E. 1996b, A\&A, 311, 927 